Y. Suyama

Nagoya Math. J.

Vol. 158 (2000), 1-42

\title{
CONFORMALLY FLAT HYPERSURFACES IN EUCLIDEAN 4-SPACE
}

\author{
YOSHIHIKO SUYAMA
}

\begin{abstract}
We study generic and conformally flat hypersurfaces in Euclidean four-space. What kind of conformally flat three manifolds are really immersed generically and conformally in Euclidean space as hypersurfaces? According to the theorem due to Cartan [1], there exists an orthogonal curvature-line coordinate system at each point of such hypersurfaces. This fact is the first step of our study. We classify such hypersurfaces in terms of the first fundamental form. In this paper, we consider hypersurfaces with the first fundamental forms of certain specific types. Then, we give a precise representation of the first and the second fundamental forms of such hypersurfaces, and give exact shapes in Euclidean space of them.
\end{abstract}

\section{$\S 1$. Introduction}

In this paper we study (topologically open, ) generic and conformally flat hypersurfaces in Euclidean 4-space $\mathbf{R}^{4}$. A smooth hypersurface in $\mathbf{R}^{4}$ is said to be generic, if the second fundamental form has three distinct eigenvalues everywhere on $M$. Our purposes are to clarify what kind of conformally flat three-manifolds are really immersed generically and conformally in $\mathbf{R}^{4}$, and to determine the shapes of their images immersed in $\mathbf{R}^{4}$.

The local theory of generic and conformally flat hypersurfaces in $\mathbf{R}^{4}$ was studied by Cartan (cf. $\S 2$ ). According to his theory there is a special local coordinate system at each point of such a hypersurface: The first and second fundamental forms are represented in diagonal forms simultaneously in the coordinate system (cf. $\S 2$ ), which we call an admissible coordinate system of conformally flat hypersurfaces in $\mathbf{R}^{4}$. The existence of admissible coordinate systems gives the first step of our study. From Cartan's another

Received October 1, 1998.

1991 Mathematics Subject Classification: Primary 53A30; Secondary 53B25, 53C40, $53 \mathrm{C} 42$.

Partly supported by the Grants-in-Aid for Scientific as well as Co-operative Research, the Ministry of Education, Science, Sports and Culture, Japan. 
result, we can know whether a generic hypersurface in $\mathbf{R}^{4}$ is conformally flat or not by examining the principal curvatures (cf. $\S 2$ ). In this paper, we give a precise representation of the first and the second fundamental forms of generic and conformally flat hypersurfaces. We also give exact shapes in $\mathbf{R}^{4}$ of such hypersurfaces.

For this purpose, we represent the first fundamental form $g$ and the second fundamental form $s$ of a generic and conformally flat hypersurface by using an admissible coordinate system $x=\left(x^{1}, x^{2}, x^{3}\right)$. Among the general form

$$
g=e^{2 P(x)}\left\{e^{2 f(x)}\left(d x^{1}\right)^{2}+e^{2 h(x)}\left(d x^{2}\right)^{2}+\left(d x^{3}\right)^{2}\right\}
$$

of the first fundamental form, we consider the following types (T.1) and (T.2) of $g($ and $s)$ :

$$
\left\{\begin{array}{l}
g=e^{2 P(x)}\left\{\left(d x^{1}\right)^{2}+\left(d x^{2}\right)^{2}+\left(d x^{3}\right)^{2}\right\} \\
s=e^{2 P(x)}\left\{\lambda(x)\left(d x^{1}\right)^{2}+\mu(x)\left(d x^{2}\right)^{2}+\nu(x)\left(d x^{3}\right)^{2}\right\}
\end{array}\right.
$$

where $P(x)=P\left(x^{1}, x^{2}, x^{3}\right)$ and $\lambda(x), \mu(x)$ and $\nu(x)$ are principal curvatures corresponding to $x^{1}$-curve, $x^{2}$-curve and $x^{3}$-curve in hypersurface, respectively.

$$
\left\{\begin{array}{l}
g=e^{2 f(x)}\left(d x^{1}\right)^{2}+e^{2 h(x)}\left(d x^{2}\right)^{2}+\left(d x^{3}\right)^{2} \\
s=e^{2 f(x)} \lambda(x)\left(d x^{1}\right)^{2}+e^{2 h(x)} \mu(x)\left(d x^{2}\right)^{2}+\nu(x)\left(d x^{3}\right)^{2}
\end{array}\right.
$$

where $f(x)=f\left(x^{1}, x^{2}, x^{3}\right)$ and $h(x)=h\left(x^{1}, x^{2}, x^{3}\right)$.

We denote $f_{i}=\partial f / \partial x^{i}$ and $f_{i j}=\partial^{2} f / \partial x^{i} \partial x^{j}$ for a smooth function $f$. We study a maximal hypersurface with metric $g$ of types (T.1) or (T.2). Here, we mean a connected hypersurface $M$ is maximal, if there is no connected hypersurface $\bar{M}$ satisfying $\bar{M} \supsetneq M$. Main results are as follows:

THEOREM 1. We assume that a conformally flat hypersurface $M$ in $\mathbf{R}^{4}$ has the first fundamental form $g$ of type (T.1), and $\lambda>\mu>\nu$ for each point of $M$. Then we have the following (1), (2) and (3).

$$
\begin{aligned}
e^{-P(x)}=C_{1} & \sin \left(\frac{\sqrt{C}}{A} x^{1}+\theta_{1}\right)+C_{2} e^{\frac{\sqrt{C-1}}{A} x^{2}}+C_{3} e^{-\frac{\sqrt{C-1}}{A} x^{2}} \\
+ & C_{4} \sin \left(\frac{\sqrt{C(C-1)}}{A} x^{3}+\theta_{2}\right) \quad \text { for }\left(x^{1}, x^{2}, x^{3}\right) \in \mathbf{R}^{3} .
\end{aligned}
$$




$$
\left\{\begin{aligned}
\lambda(x) & =\frac{A}{C-1}\left\{\left(e^{-P}\right)_{22}-\left(e^{-P}\right)_{33}\right\} \\
\mu(x) & =\frac{A}{C}\left\{\left(e^{-P}\right)_{11}-\left(e^{-P}\right)_{33}\right\} \\
\nu(x) & =A\left\{\left(e^{-P}\right)_{11}-\left(e^{-P}\right)_{22}\right\}
\end{aligned}\right.
$$

where $A, C, C_{i}(i=1,2,3,4)$ and $\theta_{i}(i=1,2)$ are constants satisfying

$$
\left\{\begin{array}{l}
A>0, C>1, C_{2}>0, C_{i} \geq 0 \quad(i=1,3,4), \\
4(C-1) C_{2} C_{3}=C C_{1}^{2}+C(C-1) C_{4}^{2}, \quad(C-1) C_{4} \neq C_{1} .
\end{array}\right.
$$

If $(C-1) C_{4}=C_{1}$ holds in Theorem 1 , then the right hand side of $e^{-P(x)}$ in (1) vanishes at $\left(\sin \left(\frac{\sqrt{C}}{A} x^{1}+\theta_{1}\right), e^{\frac{\sqrt{C-1}}{A} x^{2}}, \sin \left(\frac{\sqrt{C(C-1)}}{A} x^{3}+\theta_{2}\right)\right)=$ $\left(-1,\left(C_{1}+C_{4}\right) / 2 C_{2},-1\right)$. We discuss this case within the proof of the following corollary at $\S 3$. We have $C_{2} C_{3} \geq 0$ by the condition (3) of Theorem 1 . If $C_{3}=0$, then we have $C_{1}=C_{4}=0$. In this case, the metric $g$ is a warped product, and reduces to a special case of (T.2).

Corollary 1. We assume $C_{2} C_{3}>0$ in Theorem 1. Then we have the following:

(1) Each $x^{i}$-curve $(i=1,3)$ is a whole circle in $\mathbf{R}^{4}$.

(2) Each $x^{2}$-curve is a connected open part of circle in $\mathbf{R}^{4}$.

More precisely, we put

$$
D\left(x^{1}, x^{3}\right)=C_{1} \sin \left(\frac{\sqrt{C}}{A} x^{1}+\theta_{1}\right)+C_{4} \sin \left(\frac{\sqrt{C(C-1)}}{A} x^{3}+\theta_{2}\right)
$$

for $\left(x^{1}, x^{3}\right) \in \mathbf{R}^{2}$, then the radius $r\left(x^{1}, x^{3}\right)$ and length $L\left(x^{1}, x^{3}\right)$ of $x^{2}$-curve are given by

$$
\left\{\begin{array}{l}
r\left(x^{1}, x^{3}\right)=\frac{A}{\sqrt{C-1} \sqrt{4 C_{2} C_{3}-D^{2}\left(x^{1}, x^{3}\right)}} \\
L\left(x^{1}, x^{3}\right)=2 r\left(x^{1}, x^{3}\right)\left[\frac{\pi}{2}-\tan ^{-1}\left(\frac{D\left(x^{1}, x^{3}\right)}{\sqrt{4 C_{2} C_{3}-D^{2}\left(x^{1}, x^{3}\right)}}\right)\right],
\end{array}\right.
$$

respectively.

(3) The hypersurface $M$ collapses respectively to a point, if $x^{2}$ tends to $\pm \infty$. 
Corollary 1 implies that the hypersurface with $C_{2} C_{3}>0$ is diffeomorphic to $\mathbf{R} \times T^{2}$.

THEOREM 2. We assume that a generic and conformally flat hypersurface $M$ in $\mathbf{R}^{4}$ has the first fundamented form $g$ of type (T.2). Then we have the following:

(1) $\nu\left(x^{1}, x^{2}, x^{3}\right)=\nu\left(x^{3}\right)$, i.e., $\nu$ depends only on variable $x^{3}$. Furthermore, each $x^{3}$-curve is a plane curve in $\mathbf{R}^{4}$, and all $x^{3}$-curves are congruent in $\mathbf{R}^{4}$.

(2) If $\nu\left(x^{3}\right) \equiv 0$, then we have $g=\left(a x^{3}+b\right)^{2} g_{S}+\left(d x^{3}\right)^{2}$, where $a$ and $b$ are constants, and $g_{S}$ is a metric of surface $S$ with constant Gaussian curvature. Furthermore, each $x^{3}$-curve is a connected part of line in $\mathbf{R}^{4}$.

(3) If the set $\left\{x^{3} \in I \mid \nu\left(x^{3}\right)=0\right\}$, where $I$ is the domain of $\nu$, does not include any open interval, then $\lambda\left(x^{1}, x^{2}, x^{3}\right)=\lambda\left(x^{3}\right)$ and $\mu\left(x^{1}, x^{2}, x^{3}\right)=$ $\mu\left(x^{3}\right)$. Furthermore, we can choose an admissible coordinate system $\left(x^{1}, x^{2}, x^{3}\right)$ so that $f\left(x^{1}, x^{2}, x^{3}\right)=f\left(x^{3}\right)$ and $h\left(x^{1}, x^{2}, x^{3}\right)=h\left(x^{3}\right)$, by replacing the first coodinate system. Then, we have the following two cases (3a) or (3b):

(3a) The function $f\left(x^{3}\right)$ is not constant, but $h\left(x^{3}\right)$ is constant. Then, the hypersurface $M$ is represented as the following immersion $\Phi$ :

$$
\Phi\left(x^{1}, x^{2}, x^{3}\right)=\left[\begin{array}{cc}
A\left(x^{1}\right) & 0 \\
0 & I
\end{array}\right]\left[\begin{array}{c}
u\left(x^{3}\right) \\
0 \\
v\left(x^{3}\right) \\
0
\end{array}\right]+\left[\begin{array}{c}
0 \\
0 \\
0 \\
x^{2}
\end{array}\right]
$$

where

$$
A\left(x^{1}\right)=\left[\begin{array}{rr}
\cos x^{1} & -\sin x^{1} \\
\sin x^{1} & \cos x^{1}
\end{array}\right], \quad I=\left[\begin{array}{ll}
1 & 0 \\
0 & 1
\end{array}\right]
$$

and each surface $\left\{x^{2}=\right.$ constant $\}$ is a surface of revolution with constant Gaussian curvature.

(3b) Both $f\left(x^{3}\right)$ and $h\left(x^{3}\right)$ are non-constant functions. Then, the hypersurface $M$ is represented as the following immersion $\Phi$ :

$$
\Phi\left(x^{1}, x^{2}, x^{3}\right)=\left[\begin{array}{cc}
A\left(x^{2}\right) & 0 \\
0 & A\left(x^{1}\right)
\end{array}\right]\left[\begin{array}{c}
u\left(x^{3}\right) \\
0 \\
v\left(x^{3}\right) \\
0
\end{array}\right] .
$$


Furthermore, the plane curve $\left(u\left(x^{3}\right), v\left(x^{3}\right)\right)$ is given by

$$
\left\{\begin{array}{l}
\left(u^{\prime}\right)^{2}+\left(v^{\prime}\right)^{2}=1, \quad\left(u^{\prime \prime}, v^{\prime \prime}\right)=\nu\left(-v^{\prime}, u^{\prime}\right) \\
a^{2}\left(u^{\prime}+\nu v\right)^{2} \pm b^{2}\left(v^{\prime}-\nu u\right)^{2}=1
\end{array}\right.
$$

where $u^{\prime}=d u / d x^{3}, v^{\prime}=d v / d x^{3}, u^{\prime \prime}=d^{2} u /\left(d x^{3}\right)^{2}, v^{\prime \prime}=d^{2} v /\left(d x^{3}\right)^{2}$, a and $b$ are positive constants.

A conformally flat hypersurface in the case (2) of Theorem 2 is either a Riemannian product $\left(S \times \mathbf{R}, g_{S}+(d t)^{2}\right)$ with $S \subset \mathbf{R}^{3}$, or a cone which has the base $\left(S, g_{S}\right)$ in a sphere $\left(S^{3}, g_{S^{3}}\right)$ and the center of $S^{3}$ as the vertex (cf. [3]).

Now, we consider curves $\left(u\left(x^{3}\right), v\left(x^{3}\right)\right)$ defined by the equation (1.1) in $(3 \mathrm{~b})$. First, we consider the case $a^{2}\left(u^{\prime}+\nu v\right)^{2}+b^{2}\left(v^{\prime}-\nu u\right)^{2}=1$. Assume $b \geq a>0$, then we have two cases (1) $b \geq a>1$ and (2) $b \geq 1>a>0$. Let $\boldsymbol{e}(s)=(\cos s, \sin s)$ be a unit circle parametrized by $s$. Let $H(s)(>0)$ be a function defined by the equation

$$
\frac{d H}{d s}= \pm H \frac{\sqrt{a^{-2} \cos ^{2} s+b^{-2} \sin ^{2} s}}{\sqrt{1-\left(a^{-2} \cos ^{2} s+b^{-2} \sin ^{2} s\right)}} .
$$

Then, we define a curve $(u(s), v(s))$ by

$$
(u(s), v(s))=H(s) \boldsymbol{e}(s) .
$$

Let us define a function $x^{3}=x^{3}(s)$ by

$$
\frac{d x^{3}}{d s}=\mp \frac{H(s)}{\sqrt{1-\left(a^{-2} \cos ^{2} s+b^{-2} \sin ^{2} s\right)}} .
$$

Corollary 2. A curve $\left(u\left(x^{3}\right), v\left(x^{3}\right)\right)$ is a solution of $a^{2}\left(u^{\prime}+\nu v\right)^{2}+$ $b^{2}\left(v^{\prime}-\nu u\right)^{2}=1$ in (1.1) of (3b) if and only if a curve $\left(u\left(x^{3}\right), v\left(x^{3}\right)\right)$ is defined by (1.2), (1.3) and (1.4), where (1) $s \in \mathbf{R}$ if $b \geq a>1$, and (2) $s \in\left\{s \in \mathbf{R} \mid a^{-2} \cos ^{2} s+b^{-2} \sin ^{2} s<1\right\}$ if $b \geq 1>a>0$.

If $a=b>1$ in (1.2) and (1.3), then the curve $(u(s), v(s))$ is a logarithmic spiral. For other curves given by Corollary 2, see Figures 1 and 2 in $\S 4$. 
Next, we consider the case $a^{2}\left(u^{\prime}+\nu v\right)^{2}-b^{2}\left(v^{\prime}-\nu u\right)^{2}=1$ in (1.1). We define functions $\bar{H}=\bar{H}(s)(>0)$ and $x^{3}=x^{3}(s)$ by equations

$$
\left\{\begin{array}{l}
\frac{d \bar{H}}{d s}= \pm \frac{\bar{H} \sqrt{a^{-2} \cos ^{2} s-b^{-2} \sin ^{2} s}}{\sqrt{1-\left(a^{-2} \cos ^{2} s-b^{-2} \sin ^{2} s\right)}} \\
\frac{d x^{3}}{d s}= \pm \frac{\bar{H}}{\sqrt{1-\left(a^{-2} \cos ^{2} s-b^{-2} \sin ^{2} s\right)}}
\end{array}\right.
$$

Let us define a curve $(u(s), v(s))$ by

$$
(u(s), v(s))=\bar{H}(s) \boldsymbol{e}(s) .
$$

Corollary 3. A curve $\left(u\left(x^{3}\right), v\left(x^{3}\right)\right)$ is a solution of $a^{2}\left(u^{\prime}+\nu v\right)^{2}-$ $b^{2}\left(v^{\prime}-\nu u\right)^{2}=1$ in (1.1) of (3b) if and only if a curve $\left(u\left(x^{3}\right), v\left(x^{3}\right)\right)$ is defined by (1.5) and (1.6), where (1) $s \in\{s \in(-\pi / 2, \pi / 2)|b / a>| \tan s \mid\}$ if $a>1$, and (2) $s \in\left\{s \in(-\pi / 2, \pi / 2)|b / a>| \tan s \mid\right.$ and $a^{-2} \cos ^{2} s-$ $\left.b^{-2} \sin ^{2} s<1\right\}$ if $0<a<1$.

For curves given by Corollary 3 , see Figures 3 and 4 in $\S 4$. We note that hypersurfaces defined by curves $\left(u\left(x^{3}\right), v\left(x^{3}\right)\right)$ given by Corollaries 2 or 3 collapse to two dimension at $u\left(x^{3}\right)=0$ and $v\left(x^{3}\right)=0$.

About Theorem 1, Lancaster [4] gave a similar result. However, he showed it under the assumption for hypersurfaces to be analytic, and he did not study shapes in Euclidean space of hypersurfaces. Note that, for our case, it is sufficient that hypersurfaces are of class $C^{4}$. On other work for generic and conformally flat hyperfurfaces, there is a study for the existence of Guichard's nets due to Jeromin [2]. We refer to Lafontaine [3] and Suyama [5] for non-generic type of conformally flat hypersurfaces.

\section{$\S 2$. Theorem due to Cartan}

In this section, we summarize the local theory due to Cartan for generic and conformally flat hypersurfaces (cf. [1], [3]).

For a generic hypersurface $M$, we can choose one-forms $\alpha, \beta$ and $\gamma$ on $M$ such that the first fundamental form $g$ and the second fundamental form $s$ are represented in the following forms:

$$
g=\alpha^{2}+\beta^{2}+\gamma^{2}, \quad s=\lambda \alpha^{2}+\mu \beta^{2}+\nu \gamma^{2},
$$

where $\lambda, \mu$ and $\nu$ are principal curvatures on $M$. The Gauss equation says that the Riemannian curvature $R$ of $M$ is given by

$$
R=\lambda \mu \alpha \wedge \beta \otimes \alpha \wedge \beta+\mu \nu \beta \wedge \gamma \otimes \beta \wedge \gamma+\nu \lambda \alpha \wedge \gamma \otimes \alpha \wedge \gamma .
$$


We denote by $X_{\alpha}, X_{\beta}$ and $X_{\gamma}$ the vector fields associated with $\alpha, \beta$ and $\gamma$, respectively. We simply denote $f_{\alpha}=X_{\alpha} f, f_{\beta}=X_{\beta} f$ and $f_{\gamma}=X_{\gamma} f$ for a smooth function $f$.

THEOREM 3. (cf. [1], [3]) A generic hypersurface $M \subset \mathbf{R}^{4}$ is conformally flat if and only if the following conditions (1) and (2) hold:

$$
\begin{gathered}
d \alpha \wedge \alpha=d \beta \wedge \beta=d \gamma \wedge \gamma=0 \\
\left\{\begin{array}{c}
(\mu-\nu) \lambda_{\alpha}+(\lambda-\nu) \mu_{\alpha}+(\mu-\lambda) \nu_{\alpha}=0 \\
(\nu-\lambda) \mu_{\beta}+(\mu-\lambda) \nu_{\beta}+(\nu-\mu) \lambda_{\beta}=0 \\
(\lambda-\mu) \nu_{\gamma}+(\nu-\mu) \lambda_{\gamma}+(\lambda-\nu) \mu_{\gamma}=0
\end{array}\right.
\end{gathered}
$$

The condition (1) of Theorem 3 implies the existence of an admissible coordinate system at each point of $M$. Let $\nabla$ be the Levi-Civita connection of $g$. The Schouten tensor $S$ on $M$ is defined by $S=R i c-(r / 4) g$, where $r$ is the scalar curvature. In general, a hypersurface $M$ is conformally flat if and only if the following three conditions (a), (b) and (c) on $g$ and $s$ hold: (a) the Gauss equation. (b) the Codazzi equation. (c) $\left(\nabla_{X} S\right)(Y, Z)=$ $\left(\nabla_{Y} S\right)(X, Z)$ for any vector fields $X, Y$ and $Z$. Theorem 3 implies that the conditions (1) and (2) are equivalent to these conditions (a), (b) and (c) under the assumption for $M$ to be generic.

In the process of the proof of Theorem 3, we obtain the conditions of covariant derivatives in terms of principal curvatures (cf. [3]). Let $\nabla^{\prime}$ be the standard connection of $\mathbf{R}^{4}$, and $N$ unit vector field normal to $M$. Then we get the following:

$$
\left\{\begin{array}{l}
\nabla_{X_{\alpha}}^{\prime} X_{\alpha}=\quad \frac{\lambda_{\beta}}{\lambda-\mu} X_{\beta}+\frac{\lambda_{\gamma}}{\lambda-\nu} X_{\gamma}+\lambda N, \quad+\frac{\mu_{\gamma}}{\mu-\nu} X_{\gamma}+\mu N, \\
\nabla_{X_{\beta}}^{\prime} X_{\beta}=\frac{\mu_{\alpha}}{\mu-\lambda} X_{\alpha} \quad+\nu N, \\
\nabla_{X_{\gamma}}^{\prime} X_{\gamma}=\frac{\nu_{\alpha}}{\nu-\lambda} X_{\alpha}+\frac{\nu_{\beta}}{\nu-\mu} X_{\beta} \\
\nabla_{X_{\alpha}}^{\prime} X_{\beta}=-\frac{\lambda_{\beta}}{\lambda-\mu} X_{\alpha}, \quad \nabla_{X_{\alpha}}^{\prime} X_{\gamma}=-\frac{\lambda_{\gamma}}{\lambda-\nu} X_{\alpha}, \\
\nabla_{X_{\beta}}^{\prime} X_{\alpha}=-\frac{\mu_{\alpha}}{\mu-\lambda} X_{\beta}, \quad \nabla_{X_{\beta}}^{\prime} X_{\gamma}=-\frac{\mu_{\gamma}}{\mu-\nu} X_{\beta}, \\
\nabla_{X_{\gamma}}^{\prime} X_{\alpha}=-\frac{\nu_{\alpha}}{\nu-\lambda} X_{\gamma}, \quad \nabla_{X_{\gamma}}^{\prime} X_{\beta}=-\frac{\nu_{\beta}}{\nu-\mu} X_{\gamma} .
\end{array}\right.
$$


Note that the covariant derivatives with respect to $\nabla$ are also determined by $(2.3)$.

\section{$\S 3$. Proofs of Theorem 1 and Corollary 1}

We assume that a metric $g$ of 3 -manifold $M$ is represented as $g=$ $e^{2 P}\left\{\left(d x^{1}\right)^{2}+\left(d x^{2}\right)^{2}+\left(d x^{3}\right)^{2}\right\}$. Note that the metric $g$ is conformally flat. Then, Christoffel's symbols of the Levi-Civita connection $\nabla$ are given by

$$
\left\{\begin{array}{lll}
\Gamma_{11}^{1}=P_{1}, & \Gamma_{12}^{1}=P_{2}, \quad \Gamma_{22}^{1}=-P_{1}, \quad \Gamma_{13}^{1}=P_{3}, & \Gamma_{33}^{1}=-P_{1} \\
\Gamma_{11}^{2}=-P_{2}, \quad \Gamma_{12}^{2}=P_{1}, \quad \Gamma_{22}^{2}=P_{2}, \quad \Gamma_{23}^{2}=P_{3}, & \Gamma_{33}^{2}=-P_{2} \\
\Gamma_{11}^{3}=-P_{3}, \quad \Gamma_{13}^{3}=P_{1}, \quad \Gamma_{22}^{3}=-P_{3}, \quad \Gamma_{23}^{3}=P_{2}, & \Gamma_{33}^{3}=P_{3} \\
\Gamma_{j k}^{i}=0 \quad \text { if }(i, j, k)=\text { permutation of }(1,2,3) . &
\end{array}\right.
$$

The curvature tensor $R$ is given by

$$
\left\{\begin{array}{l}
R_{1212}=-e^{2 P}\left\{P_{11}+P_{22}+\left(P_{3}\right)^{2}\right\} \\
R_{1313}=-e^{2 P}\left\{P_{11}+P_{33}+\left(P_{2}\right)^{2}\right\} \\
R_{2323}=-e^{2 P}\left\{P_{22}+P_{33}+\left(P_{1}\right)^{2}\right\} \\
R_{1213}=e^{2 P}\left(P_{2} P_{3}-P_{23}\right) \\
R_{1223}=e^{2 P}\left(P_{13}-P_{1} P_{3}\right) \\
R_{1323}=e^{2 P}\left(P_{1} P_{2}-P_{12}\right)
\end{array}\right.
$$

We assume that the hypersurface $(M, g) \subset\left(\mathbf{R}^{4}, g_{E}\right)$ is generic, that is, $\alpha=e^{P} d x^{1}, \beta=e^{P} d x^{2}$ and $\gamma=e^{P} d x^{3}$ in (2.1).

LEMma 3.1. The following equations (1) and (2) hold:

$$
\left\{\begin{array}{l}
e^{-P} P_{1}=-\frac{\mu_{\alpha}}{\mu-\lambda}=-\frac{\nu_{\alpha}}{\nu-\lambda}, \\
e^{-P} P_{2}=-\frac{\lambda_{\beta}}{\lambda-\mu}=-\frac{\nu_{\beta}}{\nu-\mu}, \\
e^{-P} P_{3}=-\frac{\lambda_{\gamma}}{\lambda-\nu}=-\frac{\mu_{\gamma}}{\mu-\nu} .
\end{array}\right.
$$

(2) The function $e^{-P(x)}$ is a sum of one variable functions of each $x^{1}, x^{2}$ and $x^{3}$, that is, $e^{-P(x)}=U\left(x^{1}\right)+V\left(x^{2}\right)+W\left(x^{3}\right)$. 
Proof. We have $X_{\alpha}=e^{-P} \partial / \partial x^{1}, X_{\beta}=e^{-P} \partial / \partial x^{2}$ and $X_{\gamma}=$ $e^{-P} \partial / \partial x^{3}$

(1) Since

$$
\frac{\lambda_{\beta}}{\lambda-\mu} X_{\beta}+\frac{\lambda_{\gamma}}{\lambda-\nu} X_{\gamma}=e^{-P} \nabla_{\frac{\partial}{\partial x^{1}}}\left(e^{-P} \frac{\partial}{\partial x^{1}}\right)=-e^{-P} P_{2} X_{\beta}-e^{-P} P_{3} X_{\gamma}
$$

by (2.3) and (3.1), we obtain the equations $e^{-P} P_{2}=-\lambda_{\beta} /(\lambda-\mu)$ and $e^{-P} P_{3}=-\lambda_{\gamma} /(\lambda-\nu)$. The proofs of other equations of (1) are similar to the above.

(2) We have $P_{i} P_{j}-P_{i j}=0(i \neq j)$ by $(2.2)$ and $(3.2)$. Since $\left(e^{-P}\right)_{i j}=$ $e^{-P}\left(-P_{i j}+P_{i} P_{j}\right)$, we obtain $\left(e^{-P}\right)_{12}=\left(e^{-P}\right)_{13}=\left(e^{-P}\right)_{23}=0$.

LEMMA 3.2. We have the following statements (1) and (2):

(1) Each principal curvature is constant along the principal curvature line, that is, $\lambda_{\alpha}=\mu_{\beta}=\nu_{\gamma}=0$.

(2) Each principal curvature line is a part of circle or line in $\mathbf{R}^{4}$.

Proof. (1) Since $(\nu-\lambda) \mu_{\alpha}+(\lambda-\mu) \nu_{\alpha}=0$ by Lemma 3.1, we obtain $\lambda_{\alpha}=0$ by Theorem 3-(2). In the same way, we have $\mu_{\beta}=\nu_{\gamma}=0$.

(2) We prove the statement only for $x^{1}$-curve. We have

$$
\begin{aligned}
\frac{\partial}{\partial x^{1}}\left(\frac{\lambda_{\beta}}{\lambda-\mu}\right) & =\frac{\partial}{\partial x^{1}}\left(e^{-P} \frac{\lambda_{2}}{\lambda-\mu}\right)=\lambda_{2}\left\{-e^{-P} \frac{P_{1}}{\lambda-\mu}+e^{-P} \frac{\mu_{1}}{(\lambda-\mu)^{2}}\right\} \\
& =\lambda_{2}\left\{-\frac{\mu_{\alpha}}{(\lambda-\mu)^{2}}+e^{-P} \frac{\mu_{1}}{(\lambda-\mu)^{2}}\right\}=0
\end{aligned}
$$

by Lemma 3.1-(1) and $\lambda_{1}=0$. Furthermore, the equation $\frac{\partial}{\partial x^{1}}\left(\frac{\lambda_{\gamma}}{\lambda-\nu}\right)=0$ is obtained in the same way. Therefore, since

$$
\left(\nabla_{X_{\alpha}}^{\prime}\right)^{2} X_{\alpha}=-\left[\left(\frac{\lambda_{\beta}}{\lambda-\mu}\right)^{2}+\left(\frac{\lambda_{\gamma}}{\lambda-\nu}\right)^{2}+\lambda^{2}\right] X_{\alpha}
$$

and the function $\left[\left(\frac{\lambda_{\beta}}{\lambda-\mu}\right)^{2}+\left(\frac{\lambda_{\gamma}}{\lambda-\nu}\right)^{2}+\lambda^{2}\right]$ is constant along $x^{1}$-curve, each $x^{1}$-curve is a circle or a line in $\mathbf{R}^{4}$.

Remark. If $C_{3}=0$ in Theorem 1 , then $C_{1}=C_{4}=0$. In this case we have $\mu \equiv 0$ by Theorem 1-(2), that is, each $x^{2}$-curve is a part of line in $\mathbf{R}^{4}$. If $C_{2} C_{3}>0$, then each $x^{i}$-curve $(i=1,2,3)$ is a part of circle in $\mathbf{R}^{4}$. 
LEMmA 3.3. We assume $\lambda>\mu>\nu$. Then, there exists a constant $C$ $(>1)$ such that

$$
\frac{\lambda-\nu}{\lambda-\mu}=C, \frac{\lambda-\mu}{\mu-\nu}=\frac{1}{C-1} \text { and } \frac{\mu-\nu}{\lambda-\nu}=\frac{C-1}{C} .
$$

Proof. By Theorem 3-(2) and Lemma 3.2-(1), we have

$$
\begin{aligned}
& \left(\frac{\lambda-\nu}{\lambda-\mu}\right)_{1}=\frac{-\nu_{1}(\lambda-\mu)+\mu_{1}(\lambda-\nu)}{(\lambda-\mu)^{2}}=0, \\
& \left(\frac{\lambda-\nu}{\lambda-\mu}\right)_{2}=\frac{\lambda_{2}(\nu-\mu)-\nu_{2}(\lambda-\mu)}{(\lambda-\mu)^{2}}=0 \\
& \left(\frac{\lambda-\nu}{\lambda-\mu}\right)_{3}=\frac{\lambda_{3}(\nu-\mu)+\mu_{3}(\lambda-\nu)}{(\lambda-\mu)^{2}}=0 .
\end{aligned}
$$

Futhermore, since $\left(\frac{\lambda-\mu}{\lambda-\nu}\right)+\left(\frac{\mu-\nu}{\lambda-\nu}\right)=1$ and $\left(\frac{\lambda-\nu}{\mu-\nu}\right)-\left(\frac{\lambda-\mu}{\mu-\nu}\right)=1$, we have Lemma.

Let us put $\mu-\nu=Q\left(x^{1}, x^{2}, x^{3}\right)(>0)$. We have to find the function $Q ; \mu=Q+\nu, \lambda=[C /(C-1)] Q+\nu$. Since the Gauss equation implies

$$
\left\{\begin{array}{l}
\lambda \mu e^{2 P}=-\left(P_{11}+P_{22}+\left(P_{3}\right)^{2}\right) \\
\mu \nu e^{2 P}=-\left(P_{22}+P_{33}+\left(P_{1}\right)^{2}\right) \\
\nu \lambda e^{2 P}=-\left(P_{11}+P_{33}+\left(P_{2}\right)^{2}\right)
\end{array}\right.
$$

we have

$$
\left\{\begin{array}{l}
\mu(\lambda-\nu)=e^{-P}\left\{\left(e^{-P}\right)_{11}-\left(e^{-P}\right)_{33}\right\} \\
\lambda(\mu-\nu)=e^{-P}\left\{\left(e^{-P}\right)_{22}-\left(e^{-P}\right)_{33}\right\} \\
\nu(\mu-\lambda)=e^{-P}\left\{\left(e^{-P}\right)_{22}-\left(e^{-P}\right)_{11}\right\}
\end{array}\right.
$$

For a while we assume $\mu \nu \neq 0$ (see the statement before Lemma 3.6). Then, we have

$$
C=\frac{\lambda-\nu}{\lambda-\mu}=\frac{\left(e^{-P}\right)_{11}-\left(e^{-P}\right)_{33}}{\left(e^{-P}\right)_{11}-\left(e^{-P}\right)_{22}} \frac{\nu}{\mu}=\frac{\left(e^{-P}\right)_{11}-\left(e^{-P}\right)_{33}}{\left(e^{-P}\right)_{11}-\left(e^{-P}\right)_{22}} \frac{\nu}{Q+\nu} .
$$

Therefore, since

$$
Q=\frac{\nu}{C} \frac{\left\{\left(e^{-P}\right)_{11}-\left(e^{-P}\right)_{33}\right\}-C\left\{\left(e^{-P}\right)_{11}-\left(e^{-P}\right)_{22}\right\}}{\left(e^{-P}\right)_{11}-\left(e^{-P}\right)_{22}},
$$


we have

$$
\left\{\begin{array}{l}
\frac{\lambda}{\left(e^{-P}\right)_{22}-\left(e^{-P}\right)_{33}}=\frac{1}{C-1} \frac{\nu}{\left(e^{-P}\right)_{11}-\left(e^{-P}\right)_{22}}, \\
\frac{\mu}{\left(e^{-P}\right)_{11}-\left(e^{-P}\right)_{33}}=\frac{1}{C} \frac{\nu}{\left(e^{-P}\right)_{11}-\left(e^{-P}\right)_{22}} .
\end{array}\right.
$$

LEMmA 3.4. There exists a constant $A$ such that $\nu=A\left\{\left(e^{-P}\right)_{11}-\right.$ $\left.\left(e^{-P}\right)_{22}\right\}$.

Proof. Since $\lambda=\lambda\left(x^{2}, x^{3}\right), \mu=\mu\left(x^{1}, x^{3}\right), \nu=\nu\left(x^{1}, x^{2}\right)$ and $e^{-P}=$ $U\left(x^{1}\right)+V\left(x^{2}\right)+W\left(x^{3}\right)$ from Lemmas 3.1 and 3.2, the function $\nu /\left[\left(e^{-P}\right)_{11}-\right.$ $\left.\left(e^{-P}\right)_{22}\right]$ depends only on variables $x^{1}$ and $x^{2}$, i.e., $\nu /\left[\left(e^{-P}\right)_{11}-\left(e^{-P}\right)_{22}\right]=$ $A\left(x^{1}, x^{2}\right)$. Similarly, we have

$$
\frac{\lambda}{\left(e^{-P}\right)_{22}-\left(e^{-P}\right)_{33}}=\bar{A}\left(x^{2}, x^{3}\right), \quad \frac{\mu}{\left(e^{-P}\right)_{11}-\left(e^{-P}\right)_{33}}=\overline{\bar{A}}\left(x^{1}, x^{3}\right) .
$$

Therefore, we have that all $A\left(x^{1}, x^{2}\right), \bar{A}\left(x^{2}, x^{3}\right), \overline{\bar{A}}\left(x^{1}, x^{3}\right)$ are constant by $(3.5)$.

We get the following Lemma from (3.5) and Lemma 3.4.

LEMMA 3.5. We have

$$
\begin{aligned}
& \lambda=\frac{A}{C-1}\left\{\left(e^{-P}\right)_{22}-\left(e^{-P}\right)_{33}\right\}, \quad \mu=\frac{A}{C}\left\{\left(e^{-P}\right)_{11}-\left(e^{-P}\right)_{33}\right\}, \\
& \nu=A\left\{\left(e^{-P}\right)_{11}-\left(e^{-P}\right)_{22}\right\} .
\end{aligned}
$$

Since $M$ is generic, the constant $A$ is not zero. We may assume $A>0$. If $\mu=0$ or $\nu=0$, then we have $\lambda \nu \neq 0$ or $\lambda \mu \neq 0$. Therefore, even in the case $\mu=0$ or $\nu=0$, we also have Lemma 3.5 in the same way.

LEMMA 3.6. We have

$$
e^{-P}=\frac{A^{2}}{C(C-1)}\left\{-(C-1)\left(e^{-P}\right)_{11}+C\left(e^{-P}\right)_{22}-\left(e^{-P}\right)_{33}\right\} .
$$

Proof. We have $\mu(\lambda-\nu)=e^{-P}\left\{\left(e^{-P}\right)_{11}-\left(e^{-P}\right)_{33}\right\}$ by (3.4). On the other hand, we have

$$
\begin{aligned}
\mu(\lambda-\nu)= & \frac{A^{2}}{C}(C-1) \\
& \quad \times\left\{-(C-1)\left(e^{-P}\right)_{11}-\left(e^{-P}\right)_{11}+C\left(e^{-P}\right)_{22}-\left(e^{-P}\right)_{33}\right\}
\end{aligned}
$$

by Lemma 3.5. Therefore, we have Lemma. 
LEMMA 3.7. We have

$$
\begin{aligned}
e^{-P}=C_{1} & \sin \left(\frac{\sqrt{C}}{A} x^{1}+\theta_{1}\right)+C_{2} e^{\frac{\sqrt{C-1}}{A} x^{2}}+C_{3} e^{-\frac{\sqrt{C-1}}{A} x^{2}} \\
+ & C_{4} \sin \left(\frac{\sqrt{C(C-1)}}{A} x^{3}+\theta_{2}\right)
\end{aligned}
$$

where $C_{i}(i=1,2,3,4)$ and $\theta_{i}(i=1,2)$ are constant.

Proof. Since $e^{-P}=U\left(x^{1}\right)+V\left(x^{2}\right)+W\left(x^{3}\right)$, we have

$$
U=-\frac{A^{2}}{C} U_{11}, \quad V=\frac{A^{2}}{C-1} V_{22}, \quad W=-\frac{A^{2}}{C(C-1)} W_{33}
$$

by Lemma 3.6.

In Lemma 3.7, we have at least $C_{2} \geq 0$ and $C_{3} \geq 0$, because $e^{-P}>0$. Furthermore, we may assume $C_{i} \geq 0(i=1,4)$ from the representation of $e^{-P}$.

LEMMA 3.8. The constants $C_{i}(i=1,2,3,4)$ satisfy the equation

$$
4(C-1) C_{2} C_{3}=C C_{1}^{2}+C(C-1) C_{4}^{2} .
$$

Proof. We have

$$
\begin{aligned}
\lambda \mu= & e^{-P}\left[\left(e^{-P}\right)_{11}+\left(e^{-P}\right)_{22}\right]-\left[\left(e^{-P}\right)_{1}^{2}+\left(e^{-P}\right)_{2}^{2}+\left(e^{-P}\right)_{3}^{2}\right] \\
= & \frac{A^{2}}{C(C-1)}\left[-(C-1)\left(e^{-P}\right)_{11}+C\left(e^{-P}\right)_{22}-\left(e^{-P}\right)_{33}\right] \\
& \times\left[\left(e^{-P}\right)_{11}+\left(e^{-P}\right)_{22}\right]-\left[\left(e^{-P}\right)_{1}^{2}+\left(e^{-P}\right)_{2}^{2}+\left(e^{-P}\right)_{3}^{2}\right]
\end{aligned}
$$

by (3.3) and Lemma 3.6. On the other hand, we have

$$
\lambda \mu=\frac{A^{2}}{C(C-1)}\left\{\left(e^{-P}\right)_{11}-\left(e^{-P}\right)_{33}\right\}\left\{\left(e^{-P}\right)_{22}-\left(e^{-P}\right)_{33}\right\}
$$

by Lemma 3.5. Thus, we have

$$
\begin{aligned}
& \frac{A^{2}}{C(C-1)}\left[(C-1)\left(e^{-P}\right)_{11}^{2}-C\left(e^{-P}\right)_{22}^{2}+\left(e^{-P}\right)_{33}^{2}\right] \\
& =-\left[\left(e^{-P}\right)_{1}^{2}+\left(e^{-P}\right)_{2}^{2}+\left(e^{-P}\right)_{3}^{2}\right] .
\end{aligned}
$$

Finally, we have Lemma from Lemma 3.7. 
From Lemma 3.8, we have $C_{2}>0$ or $C_{3}>0$. Indeed, if $C_{2}=C_{3}=0$, then $C_{1}=C_{4}=0$ and $e^{-P} \equiv 0$. Therefore, we may assume $C_{2}>0$.

Finally, we study the condition that the right hand side of $e^{-P}$ in Lemma 3.7 becomes positive. Putting $e^{\frac{\sqrt{C-1}}{A} x^{2}}=u$, then we have $e^{-P}=$ $C_{2} u+C_{3} u^{-1}+D\left(x^{1}, x^{3}\right)$. Since

$$
\begin{aligned}
(C-1)\left[4 C_{2} C_{3}-D^{2}\left(x^{1}, x^{3}\right)\right] & \geq 4(C-1) C_{2} C_{3}-(C-1)\left(C_{1}+C_{4}\right)^{2} \\
& =\left\{C_{1}-(C-1) C_{4}\right\}^{2} \geq 0
\end{aligned}
$$

by Lemma 3.8, we have $C_{2} u+C_{3} u^{-1}+D\left(x^{1}, x^{3}\right)>0$ if $C_{1} \neq(C-1) C_{4}$. If $C_{1}=(C-1) C_{4}$, then it vanishes at $u=\left(C_{1}+C_{4}\right) / 2 C_{2}$ and $\left(\sin \left(\frac{\sqrt{C}}{A} x^{1}+\right.\right.$ $\left.\left.\theta_{1}\right), \sin \left(\frac{\sqrt{C(C-1)}}{A} x^{3}+\theta_{2}\right)\right)=(-1,-1)$. Therefore, we assumed $C_{1} \neq(C-$ 1) $C_{4}$ in Theorem 1.

Conversely, the metric $g$ and the second fundamental form $s$ given by Theorem 1 satisfy the Gauss and the Codazzi equations. Therefore, the 3 manifold is realized as a hypersurface in $\mathbf{R}^{4}$. Furthermore, since the principal curvatures $\lambda, \mu$ and $\nu$ satisfy the condition (2) of Theorem 3 , the hypersurface is conformally flat. This completes the proof of Theorem 1 .

Now, we prove Corollary 1-(1). We have

$$
\begin{aligned}
& \left(\nabla_{X_{\alpha}}^{\prime}\right)^{2} X_{\alpha}=-\left[\left(\frac{\lambda_{\beta}}{\lambda-\mu}\right)^{2}+\left(\frac{\lambda_{\gamma}}{\lambda-\nu}\right)^{2}+\lambda^{2}\right] X_{\alpha} \\
& =-\frac{1}{A^{2}}\left[(C-1)\left(C_{2} u-C_{3} u^{-1}\right)^{2}+(C-1) C C_{4}^{2} \cos ^{2}\left(\frac{\sqrt{C(C-1)}}{A} x^{3}+\theta_{2}\right)\right. \\
& \left.\quad+\left\{C_{2} u+C_{3} u^{-1}+C C_{4} \sin \left(\frac{\sqrt{C(C-1)}}{A} x^{3}+\theta_{2}\right)\right\}^{2}\right] X_{\alpha}
\end{aligned}
$$

where $u=e^{\frac{\sqrt{C-1}}{A} x^{2}}$. If $u=\sqrt{C_{3} / C_{2}}, \cos \left(\frac{\sqrt{C(C-1)}}{A} x^{3}+\theta_{2}\right)=0$ and $C_{2} u+$ $C_{3} u^{-1}-C C_{4}=0$, then $4 C_{2} C_{3}=C^{2} C_{4}^{2}$ and $\left(\nabla_{X_{\alpha}}^{\prime}\right)^{2} X_{\alpha}=0$. The condition ${ }_{4} C_{2} C_{3}=C^{2} C_{4}^{2}$ is equivalent to $C_{1}=(C-1) C_{4}$ by Lemma 3.8. We omit this case from Theorem 1 . Therefore, since the coefficient of $X_{\alpha}$ in $\left(\nabla_{X_{\alpha}}^{\prime}\right)^{2} X_{\alpha}$ is negative, each $x^{1}$-curve is a circle in $\mathbf{R}^{4}$. Similarly, each $x^{3}$-curve is a circle in $\mathbf{R}^{4}$ if $C_{1} \neq(C-1) C_{4}$. In particular, we have

$$
\left(\nabla_{X_{\gamma}}^{\prime}\right)^{2} X_{\gamma}=-\frac{1}{A^{2}}\left[C C_{1}^{2} \cos ^{2}\left(\frac{\sqrt{C}}{A} x^{1}+\theta_{1}\right)+(C-1)\left(C_{2} u-C_{3} u^{-1}\right)^{2}\right.
$$




$$
\left.+\left\{C C_{1} \sin \left(\frac{\sqrt{C}}{A} x^{1}+\theta_{1}\right)+(C-1)\left(C_{2} u+C_{3} u^{-1}\right)\right\}^{2}\right] X_{\gamma} .
$$

In the case $C_{1}=(C-1) C_{4}$, an $x^{1}$-curve for fixed $\left(x^{2}, x^{3}\right)$ satisfying $\left(e^{\frac{\sqrt{C-1}}{A} x^{2}}, \sin \left(\frac{\sqrt{C(C-1)}}{A} x^{3}+\theta_{2}\right)\right)=\left(\sqrt{C_{3} / C_{2}},-1\right)$ is a line in $\mathbf{R}^{4}$. We note $\sqrt{C_{3} / C_{2}}=\left(C_{1}+C_{4}\right) / 2 C_{2}$ in this case.

Finally, we prove Corollary 1-(2). Since

$$
\left(\nabla_{X_{\beta}}^{\prime}\right)^{2} X_{\beta}=-\left[\left(\frac{\mu_{\alpha}}{\mu-\lambda}\right)^{2}+\left(\frac{\mu_{\gamma}}{\mu-\nu}\right)^{2}+\mu^{2}\right] X_{\beta}=-\left(\frac{1}{r\left(x^{1}, x^{3}\right)}\right)^{2} X_{\beta}
$$

the radius $r\left(x^{1}, x^{3}\right)$ of an $x^{2}$-curve is

$$
r\left(x^{1}, x^{3}\right)=\frac{A}{\sqrt{C-1} \sqrt{4 C_{2} C_{3}-D^{2}\left(x^{1}, x^{3}\right)}}
$$

by Lemmas 3.6 and 3.8. The length $L\left(x^{1}, x^{3}\right)$ of an $x^{2}$-curve is

$$
\begin{aligned}
L\left(x^{1}, x^{3}\right) & =\int_{-\infty}^{\infty} e^{P\left(x^{1}, x^{2}, x^{3}\right)} d x^{2}=\int_{0}^{\infty} \frac{1}{C_{2} t^{2}+D\left(x^{1}, x^{3}\right) t+C_{3}} \frac{A}{\sqrt{C-1}} d t \\
& =2 r\left(x^{1}, x^{2}\right)\left[\frac{\pi}{2}-\tan ^{-1}\left(\frac{D\left(x^{1}, x^{3}\right)}{\sqrt{4 C_{2} C_{3}-D^{2}\left(x^{1}, x^{3}\right)}}\right)\right] .
\end{aligned}
$$

In the case $C_{1}=(C-1) C_{4}$, we have $\left(\nabla_{X_{\beta}}^{\prime}\right)^{2} X_{\beta}=0$ along an $x^{2}$-curve for fixed $\left(x^{1}, x^{3}\right)$ determined by $\left(\sin \left(\frac{\sqrt{C}}{A} x^{1}+\theta_{1}\right), \sin \left(\frac{\sqrt{C(C-1)}}{A} x^{3}+\theta_{2}\right)\right)=$ $(-1,-1)$. Therefore, the $x^{2}$-curve is a line in $\mathbf{R}^{4}$.

\section{$\S 4$. Proofs of Theorem 2 and Corollaries 2 and 3}

We assume that a metric $g$ of 3 -manifold $M$ is represented as $g=$ $e^{2 f}\left(d x^{1}\right)^{2}+e^{2 h}\left(d x^{2}\right)^{2}+\left(d x^{3}\right)^{2}$. Chistoffel's symbols of the Levi-Civita connection $\nabla$ defined from $g$ are given by

$$
\left\{\begin{array}{l}
\Gamma_{11}^{1}=f_{1}, \quad \Gamma_{12}^{1}=f_{2}, \quad \Gamma_{22}^{1}=-e^{2 h-2 f} h_{1}, \quad \Gamma_{13}^{1}=f_{3}, \\
\Gamma_{11}^{2}=-e^{2 f-2 h} f_{2}, \quad \Gamma_{12}^{2}=h_{1}, \quad \Gamma_{22}^{2}=h_{2}, \quad \Gamma_{23}^{2}=h_{3}, \\
\Gamma_{11}^{3}=-e^{2 f} f_{3}, \quad \Gamma_{22}^{3}=-e^{2 h} h_{3}, \\
\Gamma_{23}^{1}=\Gamma_{33}^{1}=\Gamma_{13}^{2}=\Gamma_{33}^{2}=\Gamma_{12}^{3}=\Gamma_{13}^{3}=\Gamma_{23}^{3}=\Gamma_{33}^{3}=0 .
\end{array}\right.
$$


The curvature tensor $R$ is given by

$$
\left\{\begin{aligned}
R_{212}^{1}= & -f_{22}-\left(f_{2}\right)^{2}+f_{2} h_{2} \\
& \quad-e^{2 h-2 f}\left(h_{11}+\left(h_{1}\right)^{2}-f_{1} h_{1}\right)-e^{2 h} f_{3} h_{3} \\
R_{223}^{1}= & e^{2 h-2 f}\left(h_{13}+h_{1} h_{3}-f_{3} h_{1}\right) \\
R_{213}^{1}=- & f_{23}-f_{2} f_{3}+f_{2} h_{3} \\
R_{313}^{1}= & -f_{33}-\left(f_{3}\right)^{2}, R_{323}^{2}=-h_{33}-\left(h_{3}\right)^{2}, R_{323}^{1}=R_{313}^{2}=0
\end{aligned}\right.
$$

Since the metric $g$ is conformally flat, we have

$$
C_{k l}^{i}=R_{k, l}^{i}-R_{l, k}^{i}-\frac{1}{4}\left(\delta_{k}^{i} r_{, l}-\delta_{l}^{i} r_{, k}\right)=0:
$$

(4.3) $C_{23}^{1}=0 \Longleftrightarrow\left\{h_{13}+h_{1} h_{3}-f_{3} h_{1}\right\}_{2}$

$$
=\left\{h_{13}+h_{1} h_{3}-f_{3} h_{1}\right\} f_{2}+\left\{f_{23}+f_{2} f_{3}-f_{2} h_{3}\right\} h_{1} .
$$

(4.4) $C_{31}^{2}=0 \Longleftrightarrow\left\{f_{23}+f_{2} f_{3}-f_{2} h_{3}\right\}_{1}$

$$
=\left\{h_{13}+h_{1} h_{3}-f_{3} h_{1}\right\} f_{2}+\left\{f_{23}+f_{2} f_{3}-f_{2} h_{3}\right\} h_{1} .
$$

(4.5) $C_{12}^{3}=0 \Longleftrightarrow\left\{f_{23}+f_{2} f_{3}-f_{2} h_{3}\right\}_{1}=\left\{h_{13}+h_{1} h_{3}-f_{3} h_{1}\right\}_{2}$.

(4.6) $C_{23}^{3}=0 \Longleftrightarrow$

$$
\begin{aligned}
\left\{e^{-2 h}\right. & \left.\left(f_{22}+\left(f_{2}\right)^{2}-f_{2} h_{2}\right)\right\}_{2}+\left\{e^{-2 f}\left(h_{11}+\left(h_{1}\right)^{2}-f_{1} h_{1}\right)\right\}_{2} \\
& \quad-\left\{f_{33}+\left(f_{3}\right)^{2}+h_{33}+\left(h_{3}\right)^{2}-f_{3} h_{3}\right\}_{2} \\
=- & 2\left\{f_{23}+f_{2} f_{3}-f_{2} h_{3}\right\}_{3}-2\left\{f_{23}+f_{2} f_{3}-f_{2} h_{3}\right\} h_{3} .
\end{aligned}
$$

(4.7) $C_{31}^{3}=0 \Longleftrightarrow$

$$
\begin{aligned}
\left\{e^{-2 h}\left(f_{22}+\left(f_{2}\right)^{2}-f_{2} h_{2}\right)\right\}_{1}+\left\{e^{-2 f}\left(h_{11}+\left(h_{1}\right)^{2}-f_{1} h_{1}\right)\right\}_{1} \\
-\left\{f_{33}+\left(f_{3}\right)^{2}+h_{33}+\left(h_{3}\right)^{2}-f_{3} h_{3}\right\}_{1} \\
=-2\left\{h_{13}+h_{1} h_{3}-f_{3} h_{1}\right\}_{3}-2\left\{h_{13}+h_{1} h_{3}-f_{3} h_{1}\right\} f_{3} .
\end{aligned}
$$

(4.8) $C_{23}^{2}=0 \Longleftrightarrow$

$$
\begin{aligned}
e^{-2 h}\left\{f_{22}\right. & \left.+\left(f_{2}\right)^{2}-f_{2} h_{2}\right\}_{3}+\left\{e^{-2 f}\left(h_{11}+\left(h_{1}\right)^{2}-f_{1} h_{1}\right)\right\}_{3} \\
& +\left\{f_{3} h_{3}+h_{33}+\left(h_{3}\right)^{2}-f_{33}-\left(f_{3}\right)^{2}\right\}_{3} \\
=2 & e^{-2 h}\left\{f_{23}+f_{2} f_{3}-f_{2} h_{3}\right\}_{2}-2 e^{-2 h}\left\{f_{23}+f_{2} f_{3}-f_{2} h_{3}\right\} h_{2} \\
& +2 e^{-2 f}\left\{h_{13}+h_{1} h_{3}-f_{3} h_{1}\right\} h_{1} \\
& -2 e^{-2 f}\left\{h_{11}+\left(h_{1}\right)^{2}-f_{1} h_{1}\right\} h_{3} \\
& +2\left\{f_{33}+\left(f_{3}\right)^{2}-f_{3} h_{3}\right\} h_{3} .
\end{aligned}
$$


Now, we assume that $(M, g)$ is a generic and conformally flat hypersurface in $\mathbf{R}^{4}$, that is, $\alpha=e^{f} d x^{1}, \beta=e^{h} d x^{2}$ and $\gamma=d x^{3}$ in (2.1).

Lemma 4.1. The principal curvature $\nu$ depends only on the variable $x^{3}$.

Proof. Since $X_{\alpha}=e^{-f} \partial / \partial x^{1}, X_{\beta}=e^{-h} \partial / \partial x^{2}$, and $X_{\gamma}=\partial / \partial x^{3}$, we have

$$
0=\Gamma_{33}^{1}=\frac{\nu_{\alpha}}{\nu-\lambda} e^{-f}=\frac{\nu_{1}}{\nu-\lambda} e^{-2 f}, \quad 0=\Gamma_{33}^{2}=\frac{\nu_{\beta}}{\nu-\mu} e^{-h}=\frac{\nu_{2}}{\nu-\mu} e^{-2 h}
$$

by (2.3) and (4.1). Thus, we have $\nu_{1}=\nu_{2}=0$.

We proved a part of the statement in Theorem 2-(1) by Lemma 4.1.

Proposition 4.1. We assume $\nu\left(x^{3}\right) \equiv 0$. Then, we have $g=\left(a x^{3}+\right.$ $b)^{2} g_{S}+\left(d x^{3}\right)^{2}$, where $a$ and $b$ are constant, and $g_{S}$ is a metric of surface $S$ with constant Gaussian curvature. Furthermore, each $x^{3}$-curve is a part of line in $\mathbf{R}^{4}$.

Proof. That each $x^{3}$-curve is a part of line follows from (2.3) and $\nu \equiv 0$. Since $(\lambda \mu)_{1}=(\lambda \mu)_{2}=(\lambda / \mu)_{3}=0$ by Theorem 3-(2), there exist functions $k\left(x^{3}\right), \ell\left(x^{1}, x^{2}\right)$ and a constant $C$ such that

$$
\lambda=C k\left(x^{3}\right) \ell\left(x^{1}, x^{2}\right), \quad \mu=\frac{k\left(x^{3}\right)}{\ell\left(x^{1}, x^{2}\right)} .
$$

Furthermore, we have

$$
f_{3}=\Gamma_{13}^{1}=-\lambda_{3} / \lambda, \quad h_{3}=\Gamma_{23}^{2}=-\mu_{3} / \mu
$$

by (2.3) and (4.1). Thus, we have

$$
f_{3}=h_{3}=-k_{3} / k,
$$

that is,

$$
e^{f\left(x^{1}, x^{2}, x^{3}\right)}=C_{1}\left(x^{1}, x^{2}\right) / k\left(x^{3}\right) \quad \text { and } \quad e^{h\left(x^{1}, x^{2}, x^{3}\right)}=C_{2}\left(x^{1}, x^{2}\right) / k\left(x^{3}\right) .
$$

On the other hand, since $\left(e^{f}\right)_{33}=\left(e^{h}\right)_{33}=0$ by (2.2) and (4.2), $e^{f}$ and $e^{h}$ are at most degree one with variable $x^{3}$. Therefore, we have $1 / k\left(x^{3}\right)=$ $a x^{3}+b$, i.e.,

$$
e^{f\left(x^{1}, x^{2}, x^{3}\right)}=C_{1}\left(x^{1}, x^{2}\right)\left(a x^{3}+b\right), \quad e^{h\left(x^{1}, x^{2}, x^{3}\right)}=C_{2}\left(x^{1}, x^{2}\right)\left(a x^{3}+b\right) .
$$


Let $S$ be a surface with local coordinate system $\left(x^{1}, x^{2}\right)$ for fixed $x^{3}$. The Gaussian curvature $K$ of $S$ with the metric $g=e^{2 f}\left(d x^{1}\right)^{2}+e^{2 h}\left(d x^{2}\right)^{2}$ is given by

$$
\begin{aligned}
K & =-e^{-f-h}\left[\frac{\partial}{\partial x^{1}}\left(e^{-f} \frac{\partial}{\partial x^{1}} e^{h}\right)+\frac{\partial}{\partial x^{2}}\left(e^{-h} \frac{\partial}{\partial x^{2}} e^{f}\right)\right] \\
& =-e^{-2 f}\left[h_{11}+\left(h_{1}\right)^{2}-f_{1} h_{1}\right]-e^{-2 h}\left[f_{22}+\left(f_{2}\right)^{2}-f_{2} h_{2}\right] .
\end{aligned}
$$

By $R_{223}^{1}=R_{213}^{1}=0,(4.2),(4.6),(4.7)$ and (4.11), we have $K_{1}=K_{2}=0$. Thus, we have $K\left(x^{1}, x^{2}, x^{3}\right)=K\left(x^{3}\right)=C /\left(a x^{3}+b\right)^{2}$.

Proposition 4.1 completes the proof of Theorem 2-(2).

From now on, we assume that the set $\left\{x^{3} \in I \mid \nu\left(x^{3}\right)=0\right\}$ does not include any open interval, where $I$ is the domain of $\nu$. Since $\nabla_{X_{\gamma}}^{\prime} X_{\gamma}=\nu N$ and $\nabla_{X_{\gamma}}^{\prime} N=-\nu X_{\gamma}$ by (2.3), each $x^{3}$-curve in $M$ is a plane curve in $\mathbf{R}^{4}$, and then all $x^{3}$-curves are congruent in $\mathbf{R}^{4}$. This fact together with Lemma 4.1 implies Theorem 2-(1).

Now, we prove Theorem 2-(3) in sequence. First, we show that a generic and conformally flat hypersurface does not exist in the case $\nu\left(x^{3}\right) \equiv c$ (non-zero constant). The proof is almost similar to one of Proposition 4.1 until a half way: We assume $\nu\left(x^{3}\right) \equiv c(\neq 0)$. Then, there exist functions $k\left(x^{3}\right), \ell\left(x^{1}, x^{2}\right)$ and a constant $C$ such that $\lambda-\nu=C k\left(x^{3}\right) \ell\left(x^{1}, x^{2}\right)$ and $\mu-\nu=k\left(x^{3}\right) / \ell\left(x^{1}, x^{2}\right)$ by Theorem 3-(2). Since $f_{3}=-\lambda_{3} /(\lambda-\nu)$ and $h_{3}=-\mu_{3} /(\mu-\nu)$ by $(2.3)$ and (4.1), we have $f_{3}=h_{3}=-k_{3} / k$. Therefore, since $\lambda \nu=R_{313}^{1}=-f_{33}-\left(f_{3}\right)^{2}$ and $\mu \nu=R_{323}^{2}=-h_{33}-\left(h_{3}\right)^{2}$ by $(2.2)$ and (4.2), we have $\lambda=\mu$ by $\nu \neq 0$. This is a contradiction to the assumption $\lambda \neq \mu$.

Therefore, our assumption that the set $\left\{x^{3} \in I \mid \nu\left(x^{3}\right)=0\right\}$ does not include any open interval is equivalent to that the set $\left\{x^{3} \in I\right.$ । $\left.\left(d \nu / d x^{3}\right)\left(x^{3}\right)=0\right\}$ does not include any open interval.

LEMMA 4.2. Let an $x^{3}$-curve be in a plane $H$. Then, both vector fields $X_{\alpha}$ and $X_{\beta}$ along the curve are constant vectors perpendicular to $H$.

Proof. The vector fields $X_{\alpha}$ and $X_{\beta}$ are perpendicular to $X_{\gamma}$ and $N$. Therefore, they are perpendicular to $H$. Furthermore, we have, by (2.3),

$$
\nabla_{\frac{\partial}{\partial x^{3}}}^{\prime} X_{\alpha}=-\frac{\nu_{\alpha}}{\nu-\lambda} X_{\gamma}=0, \quad \nabla_{\frac{\partial}{\partial x^{3}}}^{\prime} X_{\beta}=-\frac{\nu_{\beta}}{\nu-\mu} X_{\gamma}=0 .
$$


By Lemma 4.1 and Lemma 4.2, the immersion $\Phi$ which represent the hypersurface $M$ is given as follows: There exist a plane $H_{0}$ in $\mathbf{R}^{4}$, a curve $P\left(x^{3}\right)$ in $H_{0}$ with arc-length parameter $x^{3}$, a map $A:\left(x^{1}, x^{2}\right) \mapsto A\left(x^{1}, x^{2}\right) \in$ $S O(4)$ with $A(0,0)=E$ and a map a : $\left(x^{1}, x^{2}\right) \mapsto \mathbf{a}\left(x^{1}, x^{2}\right) \in \mathbf{R}^{4}$ with $\mathbf{a}(0,0)=\mathbf{0}$ such that

$$
M: \Phi\left(x^{1}, x^{2}, x^{3}\right)=A\left(x^{1}, x^{2}\right) P\left(x^{3}\right)+\mathbf{a}\left(x^{1}, x^{2}\right) .
$$

Furthermore, both vector fields $X_{\alpha}$ and $X_{\beta}$,

$$
\begin{aligned}
& X_{\alpha}\left(x^{1}, x^{2}\right)=e^{-f}\left\{A\left(\dot{x}^{1}, x^{2}\right) P\left(x^{3}\right)+\mathbf{a}\left(\dot{x}^{1}, x^{2}\right)\right\}, \\
& X_{\beta}\left(x^{1}, x^{2}\right)=e^{-h}\left\{A\left(x^{1}, \dot{x}^{2}\right) P\left(x^{3}\right)+\mathbf{a}\left(x^{1}, \dot{x}^{2}\right)\right\},
\end{aligned}
$$

do not depend on variable $x^{3}$, and they are perpendicular to the plane $A\left(x^{1}, x^{2}\right) H_{0}+\mathbf{a}\left(x^{1}, x^{2}\right)$, where $A\left(\dot{x}^{1}, x^{2}\right)=\partial A\left(x^{1}, x^{2}\right) / \partial x^{1}$ and $A\left(x^{1}, \dot{x}^{2}\right)=$ $\partial A\left(x^{1}, x^{2}\right) / \partial x^{2}$.

Proposition 4.2. If there exists a point $\left(a^{1}, a^{2}\right)$ such that $\left.A\left(\dot{a}^{1}, a^{2}\right)\right|_{H_{0}}$ $=\left.A\left(a^{1}, \dot{a}^{2}\right)\right|_{H_{0}}=0$, then we have $\nu \equiv 0$, where $\left.A\left(\dot{a}^{1}, a^{2}\right)\right|_{H_{0}}$ denotes the action of $A\left(\dot{a}^{1}, a^{2}\right)$ on $H_{0}$.

Proof. Since $\left(e^{f} X_{\alpha}\right)\left(a^{1}, a^{2}, x^{3}\right)=\mathbf{a}\left(\dot{a}^{1}, a^{2}\right)$ and $\left(e^{h} X_{\beta}\right)\left(a^{1}, a^{2}, x^{3}\right)=$ $\mathbf{a}\left(a^{1}, \dot{a}^{2}\right)$, we have $e^{f}\left(a^{1}, a^{2}, x^{3}\right)=\left\|\mathbf{a}\left(\dot{a}^{1}, a^{2}\right)\right\|$ and $e^{h}\left(a^{1}, a^{2}, x^{3}\right)=\left\|\mathbf{a}\left(a^{1}, \dot{a}^{2}\right)\right\|$. Therefore, $f_{3}\left(a^{1}, a^{2}, x^{3}\right)=h_{3}\left(a^{1}, a^{2}, x^{3}\right)=0$. On the other hand, since $\nu \lambda=R_{313}^{1}=-f_{33}-\left(f_{3}\right)^{2}=0$ and $\mu \nu=R_{323}^{2}=-h_{33}-\left(h_{3}\right)^{2}=0$ for $\left(a^{1}, a^{2}, x^{3}\right)$ by $(2.2)$ and (4.2), we have either $\lambda=\mu=0$ or $\nu=0$ for $\left(a^{1}, a^{2}, x^{3}\right)$. This implies $\nu \equiv 0$ by the assumption $\lambda \neq \mu$.

We may assume that there exists a point $\left(a^{1}, a^{2}\right)$ such that $\left.A\left(\dot{a}^{1}, a^{2}\right)\right|_{H_{0}}$ $\neq 0$ by Proposition 4.2 and the assumption for $\nu$. Let us put $U=\{x=$ $\left.\left(x^{1}, x^{2}, x^{3}\right) \in M\left|A\left(\dot{x}^{1}, x^{2}\right)\right|_{H_{0}} \neq 0\right\}$. We assume that $U$ is connected. For a while, we restrict our discussion only on $U$ (cf. Proposition 4.5 below). We simply denote $x^{3}$ by $t$. Let us put $\dot{P}(t)=\boldsymbol{e}_{1}(t)$ and $\ddot{P}(t)=\nu(t) \boldsymbol{e}_{2}(t)$, where $\dot{P}(t)=d P / d t$ and $\ddot{P}(t)=d^{2} P / d t^{2}$. Then, we may assume that frames $\left\{\boldsymbol{e}_{1}(t), \boldsymbol{e}_{2}(t)\right\}$ determine an orientation of the plane $H_{0}$ so that the Gauss map $\boldsymbol{e}_{1}(t)$ moves to positive direction if $\nu$ is positive.

Lemma 4.3. Let $\left(x^{1}, x^{2}, t\right) \in U$. We define orthonormal frames $\left\{\boldsymbol{b}\left(x^{1}, x^{2}, t\right), \boldsymbol{c}\left(x^{1}, x^{2}, t\right)\right\}$ of $H_{0}$ along the curve $P(t)$ for any $\left(x^{1}, x^{2}\right)$ by

$$
\boldsymbol{b}\left(x^{1}, x^{2}, t\right)=\frac{-\lambda \boldsymbol{e}_{1}-f_{3} \boldsymbol{e}_{2}}{\left[f_{3}^{2}+\lambda^{2}\right]^{1 / 2}}\left(x^{1}, x^{2}, t\right), \boldsymbol{c}\left(x^{1}, x^{2}, t\right)=\frac{f_{3} \boldsymbol{e}_{1}-\lambda \boldsymbol{e}_{2}}{\left[f_{3}^{2}+\lambda^{2}\right]^{1 / 2}}\left(x^{1}, x^{2}, t\right) .
$$


Let us define a positive function $s\left(x^{1}, x^{2}, t\right)$ by

$$
s^{2}\left(x^{1}, x^{2}, t\right)=\left[\left(e^{f} f_{3}\right)^{2}+\left(e^{f} \lambda\right)^{2}\right]\left(x^{1}, x^{2}, t\right) .
$$

Then, we have

$$
\left\{\begin{array}{l}
A\left(\dot{x}^{1}, x^{2}\right) \boldsymbol{b}\left(x^{1}, x^{2}, t\right)=0 \\
A\left(\dot{x}^{1}, x^{2}\right) \boldsymbol{c}\left(x^{1}, x^{2}, t\right)=s\left(x^{1}, x^{2}, t\right) X_{\alpha}\left(x^{1}, x^{2}\right) .
\end{array}\right.
$$

In particular, the vector fields $\boldsymbol{b}, \boldsymbol{c}$ and the function $s$ are independent of $t$, i.e., $\boldsymbol{b}\left(x^{1}, x^{2}, t\right)=\boldsymbol{b}\left(x^{1}, x^{2}\right), \boldsymbol{c}\left(x^{1}, x^{2}, t\right)=\boldsymbol{c}\left(x^{1}, x^{2}\right)$ and $s\left(x^{1}, x^{2}, t\right)=$ $s\left(x^{1}, x^{2}\right)$.

Proof. Since

$$
\left\{\begin{array}{l}
e^{-f} A\left(\dot{x}^{1}, x^{2}\right) \boldsymbol{e}_{1}=\nabla_{X_{\alpha}}^{\prime} X_{\gamma}=-\frac{\lambda_{\gamma}}{\lambda-\nu} X_{\alpha}=f_{3} X_{\alpha} \\
e^{-f} A\left(\dot{x}^{1}, x^{2}\right) \boldsymbol{e}_{2}=\nabla_{X_{\alpha}}^{\prime} N=-\lambda X_{\alpha}
\end{array}\right.
$$

by (2.3), (4.1) and (4.12), we have (4.13). Then, the function $s$ is positive by $\left.A\left(\dot{x}^{1}, x^{2}\right)\right|_{H_{0}} \neq 0$. In particular, vector fields $\boldsymbol{b}, \boldsymbol{c}$ and the function $s$ are independent of $t$, because $A\left(\dot{x}^{1}, x^{2}\right)$ and $X_{\alpha}\left(x^{1}, x^{2}\right)$ do not depend on $t$.

For each $\left(x^{1}, x^{2}\right)$, we represent the curve $P(t)$ in terms of basis $\left\{\boldsymbol{b}\left(x^{1}, x^{2}\right), \boldsymbol{c}\left(x^{1}, x^{2}\right)\right\}: P(t)=u\left(x^{1}, x^{2}, t\right) \boldsymbol{b}\left(x^{1}, x^{2}\right)+v\left(x^{1}, x^{2}, t\right) \boldsymbol{c}\left(x^{1}, x^{2}\right)$.

Proposition 4.3. Let $\left(x^{1}, x^{2}, t\right) \in U$. There exists a function $\sigma=$ $\sigma\left(x^{1}, x^{2}\right)$ such that

$$
\begin{gathered}
\left\{\begin{array}{l}
\left(e^{f}\right)_{3}\left(x^{1}, x^{2}, t\right)=s\left(x^{1}, x^{2}\right) \cos \left[\int_{t_{0}}^{t} \nu(t) d t+\sigma\left(x^{1}, x^{2}\right)\right] \\
\left(\lambda e^{f}\right)\left(x^{1}, x^{2}, t\right)=s\left(x^{1}, x^{2}\right) \sin \left[\int_{t_{0}}^{t} \nu(t) d t+\sigma\left(x^{1}, x^{2}\right)\right],
\end{array}\right. \\
\left\{\begin{array}{l}
u\left(x^{1}, x^{2}, \dot{t}\right)=-\sin \left[\int_{t_{0}}^{t} \nu(t) d t+\sigma\left(x^{1}, x^{2}\right)\right] \\
v\left(x^{1}, x^{2}, \dot{t}\right)=\cos \left[\int_{t_{0}}^{t} \nu(t) d t+\sigma\left(x^{1}, x^{2}\right)\right]
\end{array}\right. \\
v\left(x^{1}, x^{2}, t\right)=\frac{1}{\lambda\left(x^{1}, x^{2}, t\right)} \sin \left[\int_{t_{0}}^{t} \nu(t) d t+\sigma\left(x^{1}, x^{2}\right)\right]
\end{gathered}
$$

Furthermore, the vector $\mathbf{a}\left(x^{1}, x^{2}\right)$ at (4.12) is independent of $x^{1}$, i.e., $\mathbf{a}\left(x^{1}, x^{2}\right)=\mathbf{a}\left(x^{2}\right)$. 
The proof of Proposition 4.3 is divided into Lemmas 4.4, 4.5, 4.6 and 4.7 below.

Lemma 4.4. We define a function $c\left(x^{1}, x^{2}\right)$ by $c\left(x^{1}, x^{2}\right)=e^{f}\left(x^{1}, x^{2}, t\right)-$ $s\left(x^{1}, x^{2}\right) v\left(x^{1}, x^{2}, t\right)$. Then, we have $\mathbf{a}\left(\dot{x}^{1}, x^{2}\right)=c\left(x^{1}, x^{2}\right) X_{\alpha}\left(x^{1}, x^{2}\right)$.

Proof. We have

$$
\begin{aligned}
e^{f}\left(x^{1}, x^{2}, t\right) X_{\alpha}\left(x^{1}, x^{2}\right) & =A\left(\dot{x}^{1}, x^{2}\right) P(t)+\mathbf{a}\left(\dot{x}^{1}, x^{2}\right) \\
& =s\left(x^{1}, x^{2}\right) v\left(x^{1}, x^{2}, t\right) X_{\alpha}\left(x^{1}, x^{2}\right)+\mathbf{a}\left(\dot{x}^{1}, x^{2}\right) .
\end{aligned}
$$

Furthermore, since both vectors $X_{\alpha}$ and $\mathbf{a}\left(\dot{x}^{1}, x^{2}\right)$ are independent of variable $t$, so is the function $c$.

Lemma 4.5. There exists a function $\sigma=\sigma\left(x^{1}, x^{2}\right)$ such that

$$
\left\{\begin{aligned}
\left(e^{f}\right)_{3}\left(x^{1}, x^{2}, t\right) & =\left(e^{f} f_{3}\right)\left(x^{1}, x^{2}, t\right) \\
& =s\left(x^{1}, x^{2}\right) \cos \left[\int_{t_{0}}^{t} \nu(t) d t+\sigma\left(x^{1}, x^{2}\right)\right], \\
\left(\lambda e^{f}\right)\left(x^{1}, x^{2}, t\right) & =s\left(x^{1}, x^{2}\right) \sin \left[\int_{t_{0}}^{t} \nu(t) d t+\sigma\left(x^{1}, x^{2}\right)\right] .
\end{aligned}\right.
$$

Proof. We have

$$
\left\{\begin{array}{l}
\left(e^{f} f_{3}\right)_{3}=e^{f}\left[f_{33}+f_{3}^{2}\right]=-\nu\left(\lambda e^{f}\right), \\
\left(\lambda e^{f}\right)_{3}=e^{f}\left(\lambda_{3}+\lambda f_{3}\right)=e^{f}\left[-(\lambda-\nu) f_{3}+\lambda f_{3}\right]=\nu\left(f_{3} e^{f}\right) .
\end{array}\right.
$$

Indeed, the first equation follows from (2.2) and (4.2), and the second follows from (2.3) and (4.1). Furthermore, since $s^{2}\left(x^{1}, x^{2}\right)\left(=\left(e^{f} f_{3}\right)^{2}\left(x^{1}, x^{2}, t\right)+\right.$ $\left.\left(\lambda e^{f}\right)^{2}\left(x^{1}, x^{2}, t\right)\right)$ is independent of $t$, we have Lemma.

Lemma 4.6. We have

$$
\begin{aligned}
& u\left(x^{1}, x^{2}, \dot{t}\right)=-\sin \left[\int_{t_{0}}^{t} \nu(t) d t+\sigma\left(x^{1}, x^{2}\right)\right], \\
& v\left(x^{1}, x^{2}, \dot{t}\right)=\cos \left[\int_{t_{0}}^{t} \nu(t) d t+\sigma\left(x^{1}, x^{2}\right)\right] .
\end{aligned}
$$

Proof. Differentiating the function $c$ in Lemma 4.4 with respect to $t$, we have $\left(e^{f} f_{3}\right)\left(x^{1}, x^{2}, t\right)=s\left(x^{1}, x^{2}\right) v\left(x^{1}, x^{2}, \dot{t}\right)$. Thus, we get the second equation by Lemma 4.5. Since $t$ is an arc-length parameter and the Gauss map $\left(u\left(x^{1}, x^{2}, \dot{t}\right), v\left(x^{1}, x^{2}, \dot{t}\right)\right)$ for fixed $\left(x^{1}, x^{2}\right)$ moves to positive direction if $\nu$ is positive, we have Lemma. 
LEMMA 4.7. We have

$$
v\left(x^{1}, x^{2}, t\right)=\frac{1}{\lambda\left(x^{1}, x^{2}, t\right)} \sin \left[\int_{t_{0}}^{t} \nu(t) d t+\sigma\left(x^{1}, x^{2}\right)\right]-\frac{c\left(x^{1}, x^{2}\right)}{s\left(x^{1}, x^{2}\right)} .
$$

Proof. We have

$$
v=\frac{e^{f}}{s}-\frac{c}{s}=\frac{1}{\lambda} \sin \left[\int_{t_{0}}^{t} \nu(t) d t+\sigma\left(x^{1}, x^{2}\right)\right]-\frac{c}{s}
$$

by Lemmas 4.4 and 4.5 .

We continue the proof of Proposition 4.3. We may assume $\mathbf{a}\left(\dot{x}^{1}, x^{2}\right)=0$, i.e., $c\left(x^{1}, x^{2}\right)=0$. Indeed, since

$$
\begin{aligned}
\Phi\left(\dot{x}^{1}, x^{2}, t\right) & =A\left(\dot{x}^{1}, x^{2}\right) P(t)+\mathbf{a}\left(\dot{x}^{1}, x^{2}\right)=(s v+c) X_{\alpha} \\
& =\left(\frac{s}{\lambda} \sin \left[\int_{t_{0}}^{t} \nu(t) d t+\sigma\right]-c+c\right) X_{\alpha} \\
& =\frac{s}{\lambda} \sin \left[\int_{t_{0}}^{t} \nu(t) d t+\sigma\right] X_{\alpha}
\end{aligned}
$$

$\Phi\left(\dot{x}^{1}, x^{2}, t\right)$ depends only on $(s / \lambda) \sin \left[\int_{t_{0}}^{t} \nu(t) d t+\sigma\right]$. This completes the proof of Proposition 4.3.

Proposition 4.4. Let $\left(x^{1}, x^{2}, t\right) \in U$. Then, we have the following statements:

(1) The function $\sigma=\sigma\left(x^{1}, x^{2}\right)$ in Proposition 4.3 is constant.

(2) The principal curvatures $\lambda, \mu$ and functions $u, v$ depend only on variable $t$, i.e., $\lambda\left(x^{1}, x^{2}, t\right)=\lambda(t), \mu\left(x^{1}, x^{2}, t\right)=\mu(t), u\left(x^{1}, x^{2}, t\right)=u(t)$, $v\left(x^{1}, x^{2}, t\right)=v(t)$.

(3) The frames $\left\{\boldsymbol{b}\left(x^{1}, x^{2}\right), \boldsymbol{c}\left(x^{1}, x^{2}\right)\right\}$ of $H_{0}$ do not depend on $\left(x^{1}, x^{2}\right)$, i.e., $\boldsymbol{b}\left(x^{1}, x^{2}\right)=\boldsymbol{b}$ and $\boldsymbol{c}\left(x^{1}, x^{2}\right)=\boldsymbol{c}$.

The proof of Proposition 4.4 is divided into Lemmas 4.8, 4.9 and 4.10 below.

LEMMA 4.8. We have

$$
\left\{\begin{array}{l}
v\left(\dot{x}^{1}, x^{2}, t\right)=u\left(x^{1}, x^{2}, t\right) \sigma\left(\dot{x}^{1}, x^{2}\right), \\
v\left(x^{1}, \dot{x}^{2}, t\right)=u\left(x^{1}, x^{2}, t\right) \sigma\left(x^{1}, \dot{x}^{2}\right) \\
u\left(\dot{x}^{1}, x^{2}, t\right)=-v\left(x^{1}, x^{2}, t\right) \sigma\left(\dot{x}^{1}, x^{2}\right) \\
u\left(x^{1}, \dot{x}^{2}, t\right)=-v\left(x^{1}, x^{2}, t\right) \sigma\left(x^{1}, \dot{x}^{2}\right) .
\end{array}\right.
$$


Proof. We have

$$
\begin{aligned}
v\left(\dot{x}^{1}, x^{2}, t\right) & =\frac{\partial}{\partial x^{1}}\langle\boldsymbol{c}, P(t)\rangle=\left\langle\nabla_{\frac{\partial}{\partial x^{1}}}^{\prime} \boldsymbol{c}\left(x^{1}, x^{2}\right), P(t)\right\rangle \\
& =\left\langle\nabla_{\frac{\partial}{\partial x^{1}}}^{\prime} \boldsymbol{c}, u \boldsymbol{b}+v \boldsymbol{c}\right\rangle=u\left\langle\nabla_{\frac{\partial}{\partial x^{1}}}^{\prime} \boldsymbol{c}, \boldsymbol{b}\right\rangle .
\end{aligned}
$$

On the other hand, we have

$$
\begin{aligned}
\nabla_{\frac{\partial}{\partial x^{1}}}^{\prime} \boldsymbol{c} & =\nabla_{\frac{\partial}{\partial x^{1}}}^{\prime}\left(\frac{f_{3} \boldsymbol{e}_{1}-\lambda \boldsymbol{e}_{2}}{\left[f_{3}^{2}+\lambda^{2}\right]^{1 / 2}}\right)=\frac{\lambda_{1} f_{3}-\lambda f_{13}}{f_{3}^{2}+\lambda^{2}} \boldsymbol{b} \\
& =\left(\frac{\lambda}{f_{3}}\right)_{1} \frac{\left(e^{f} f_{3}\right)^{2}}{\left(e^{f} f_{3}\right)^{2}+\left(e^{f} \lambda\right)^{2}} \boldsymbol{b} \\
& =\left(\tan \left[\int_{t_{0}}^{t} \nu(t) d t+\sigma\right]\right)_{1} \cos ^{2}\left[\int_{t_{0}}^{t} \nu(t) d t+\sigma\right] \boldsymbol{b} \\
& =\sigma\left(\dot{x}^{1}, x^{2}\right) \boldsymbol{b}
\end{aligned}
$$

Thus, we have $v\left(\dot{x}^{1}, x^{2}, t\right)=u\left(x^{1}, x^{2}, t\right) \sigma\left(\dot{x}^{1}, x^{2}\right)$. Other equations are obtained in the same way.

If $\sigma\left(x^{1}, x^{2}\right)$ is a constant function, then we have $u\left(x^{1}, x^{2}, t\right)=u(t)$, $v\left(x^{1}, x^{2}, t\right)=v(t), \boldsymbol{b}\left(x^{1}, x^{2}\right)=\boldsymbol{b}$ and $\boldsymbol{c}\left(x^{1}, x^{2}\right)=\boldsymbol{c}$ from Lemma 4.8 and its proof. Then, from $v(t)=\lambda^{-1} \sin \left[\int_{t_{0}}^{t} \nu(t) d t+\sigma\right]$ in Proposition 4.3, we also have $\lambda\left(x^{1}, x^{2}, t\right)=\lambda(t)$. Furthermore, since $(\lambda(t)-\nu(t)) \mu_{i}=0$ $(i=1,2)$ by Theorem $3-(2)$, we have $\mu\left(x^{1}, x^{2}, t\right)=\mu(t)$. Therefore, to get Proposition 4.4, we have only to prove that $\sigma\left(x^{1}, x^{2}\right)$ is constant. We set

$$
\begin{aligned}
\mathbf{R}^{4} & =\left\{X \boldsymbol{f}_{1}+Y \boldsymbol{f}_{2}+Z \boldsymbol{f}_{3}+W \boldsymbol{f}_{4}={ }^{t}(X, Y, Z, W) \mid X, Y, Z, W \in \mathbf{R}\right\}, \\
H_{0} & =\left\{X \boldsymbol{f}_{1}+Y \boldsymbol{f}_{2}={ }^{t}(X, Y, 0,0) \mid X, Y \in \mathbf{R}\right\} .
\end{aligned}
$$

Let $P(t)={ }^{t}(u(t), v(t), 0,0)$ be a curve in $H_{0}$ with arc-length parameter satisfying $(\ddot{u}, \ddot{v})(t)=\nu(t)(-\dot{v}, \dot{u})(t)$. Then, we have $M: \Phi\left(x^{1}, x^{2}, t\right)=$ $A\left(x^{1}, x^{2}\right) P(t)+\mathbf{a}\left(x^{2}\right)$ and

$$
\left\{\begin{aligned}
N\left(x^{1}, x^{2}, t\right) & =A\left(x^{1}, x^{2}\right)\left(-\dot{v}(t) \boldsymbol{f}_{1}+\dot{u}(t) \boldsymbol{f}_{2}\right) \\
X_{\alpha}\left(x^{1}, x^{2}\right) & =\left(e^{-f}\right)\left(x^{1}, x^{2}, t\right) \partial / \partial x^{1} \\
& =\left(e^{-f}\right)\left(x^{1}, x^{2}, t\right) A\left(\dot{x}^{1}, x^{2}\right) P(t)
\end{aligned}\right.
$$

by Lemma 4.2, (4.12) and Proposition 4.3. From $\nu=\nu(t)$ and Theorem 3 (2), there exists a function $q(t)$ such that

$$
\left(\lambda\left(x^{1}, x^{2}, t\right)-\nu(t)\right)\left(\mu\left(x^{1}, x^{2}, t\right)-\nu(t)\right)=q(t) .
$$


LEMMA 4.9. We have the following:

$$
\lambda\left(x^{1}, x^{2}, t\right)=\frac{\dot{v}(t) \sin \sigma\left(x^{1}, x^{2}\right)-\dot{u}(t) \cos \sigma\left(x^{1}, x^{2}\right)}{u(t) \sin \sigma\left(x^{1}, x^{2}\right)+v(t) \cos \sigma\left(x^{1}, x^{2}\right)}
$$

(2) For each $\left(x^{1}, x^{2}\right)$, there exists $t$ such that $\lambda\left(x^{1}, x^{2}, t\right) \neq 0$.

Proof. (1) We consider the action of $A^{-1}\left(x^{1}, x^{2}\right) A\left(\dot{x}^{1}, x^{2}\right)$ on $H_{0}$ for each $\left(x^{1}, x^{2}\right)$, and denote it by $\left.A^{-1}\left(x^{1}, x^{2}\right) A\left(\dot{x}^{1}, x^{2}\right)\right|_{H_{0}}$. For this purpose, we put

$$
\begin{aligned}
& H_{0} \subset \mathbf{R}^{3}=\left\{X \boldsymbol{f}_{1}+Y \boldsymbol{f}_{2}+Z \boldsymbol{f}_{3}={ }^{t}(X, Y, Z) \mid X, Y, Z \in \mathbf{R}\right\}, \\
& B(x)=\left[\begin{array}{ccc}
1 & 0 & 0 \\
0 & \cos x & -\sin x \\
0 & \sin x & \cos x
\end{array}\right], \\
& C\left(x^{1}, x^{2}\right)=\left[\begin{array}{ccc}
\cos \sigma\left(x^{1}, x^{2}\right) & -\sin \sigma\left(x^{1}, x^{2}\right) & 0 \\
\sin \sigma\left(x^{1}, x^{2}\right) & \cos \sigma\left(x^{1}, x^{2}\right) & 0 \\
0 & 0 & 0
\end{array}\right] .
\end{aligned}
$$

If we identify $\boldsymbol{b}\left(x^{1}, x^{2}\right)=\boldsymbol{f}_{1}, \boldsymbol{c}\left(x^{1}, x^{2}\right)=\boldsymbol{f}_{2}$ and $A^{-1}\left(x^{1}, x^{2}\right) X_{\alpha}\left(x^{1}, x^{2}\right)=$ $\boldsymbol{f}_{3}$, then we have $\left.A^{-1}\left(x^{1}, x^{2}\right) A\left(x^{1}, x^{2}\right)\right|_{H_{0}}=\left.s\left(x^{1}, x^{2}\right) \dot{B}(0)\right|_{H_{0}}$ by Lemma 4.3. However, since

$$
\begin{aligned}
& \boldsymbol{b}\left(x^{1}, x^{2}\right)=\cos \sigma\left(x^{1}, x^{2}\right) \boldsymbol{b}(0,0)-\sin \sigma\left(x^{1}, x^{2}\right) \boldsymbol{c}(0,0), \\
& \boldsymbol{c}\left(x^{1}, x^{2}\right)=\sin \sigma\left(x^{1}, x^{2}\right) \boldsymbol{b}(0,0)+\cos \sigma\left(x^{1}, x^{2}\right) \boldsymbol{c}(0,0)
\end{aligned}
$$

by the proof of Lemma 4.8, we have

$$
\left.A^{-1}\left(x^{1}, x^{2}\right) A\left(\dot{x}^{1}, x^{2}\right)\right|_{H_{0}}=\left.s\left(x^{1}, x^{2}\right) \dot{B}(0) C\left(x^{1}, x^{2}\right)\right|_{H_{0}}
$$

by taking $\boldsymbol{b}(0,0)=\boldsymbol{f}_{1}, \boldsymbol{c}(0,0)=\boldsymbol{f}_{2}$ (i.e., $\sigma(0,0)=0$ ) and identifying $A^{-1}\left(x^{1}, x^{2}\right) X_{\alpha}\left(x^{1}, x^{2}\right)=\boldsymbol{f}_{3}$ for each $\left(x^{1}, x^{2}\right)$. Since $X_{\alpha}=e^{-f} \partial \Phi / \partial x^{1}$ and $-\lambda X_{\alpha}=\nabla_{X_{\alpha}}^{\prime} N$, we have

$$
\begin{aligned}
& \left(e^{f}\right)\left(x^{1}, x^{2}, t\right) A^{-1}\left(x^{1}, x^{2}\right) X_{\alpha}\left(x^{1}, x^{2}\right)=A^{-1}\left(x^{1}, x^{2}\right) A\left(\dot{x}^{1}, x^{2}\right) P(t) \\
& \quad=s\left(x^{1}, x^{2}\right) \dot{B}(0) C\left(x^{1}, x^{2}\right) P(t)=s\left(x^{1}, x^{2}\right) \dot{B}(0) C\left(x^{1}, x^{2}\right)\left(u(t) \boldsymbol{f}_{1}+v(t) \boldsymbol{f}_{2}\right) \\
& \quad=s\left(x^{1}, x^{2}\right)\left\{u(t) \sin \sigma\left(x^{1}, x^{2}\right)+v(t) \cos \sigma\left(x^{1}, x^{2}\right)\right\} \boldsymbol{f}_{3},
\end{aligned}
$$


and

$$
\begin{aligned}
- & \left(\lambda e^{f}\right)\left(x^{1}, x^{2}, t\right) A^{-1}\left(x^{1}, x^{2}\right) X_{\alpha}\left(x^{1}, x^{2}\right) \\
& =e^{f}\left(x^{1}, x^{2}, t\right) A^{-1}\left(x^{1}, x^{2}\right)\left(\nabla_{X_{\alpha}}^{\prime} N\right)\left(x^{1}, x^{2}, t\right) \\
& =A^{-1}\left(x^{1}, x^{2}\right) A\left(\dot{x}^{1}, x^{2}\right)\left(-\dot{v}(t) \boldsymbol{f}_{1}+\dot{u}(t) \boldsymbol{f}_{2}\right) \\
& =s\left(x^{1}, x^{2}\right) \dot{B}(0) C\left(x^{1}, x^{2}\right)\left(-\dot{v}(t) \boldsymbol{f}_{1}+\dot{u}(t) \boldsymbol{f}_{2}\right) \\
& =s\left(x^{1}, x^{2}\right)\left\{-\dot{v}(t) \sin \sigma\left(x^{1}, x^{2}\right)+\dot{u}(t) \cos \sigma\left(x^{1}, x^{2}\right)\right\} \boldsymbol{f}_{3}
\end{aligned}
$$

by (4.14) and (4.16). Therefore, we have

$$
\lambda\left(x^{1}, x^{2}, t\right)=\frac{\dot{v}(t) \sin \sigma\left(x^{1}, x^{2}\right)-\dot{u}(t) \cos \sigma\left(x^{1}, x^{2}\right)}{u(t) \sin \sigma\left(x^{1}, x^{2}\right)+v(t) \cos \sigma\left(x^{1}, x^{2}\right)} .
$$

(2) If $\lambda\left(a^{1}, a^{2}, t\right) \equiv 0$ for some $\left(a^{1}, a^{2}\right)$, then $(u(t), v(t))$ is a line in $H_{0}$ by (1), which contradicts the assumption for $\nu$.

LEMmA 4.10. The function $\sigma\left(x^{1}, x^{2}\right)$ is constant.

Proof. First we note that, if $\mu \equiv 0$, then we have $\lambda\left(x^{1}, x^{2}, t\right)=\lambda(t)$ by (4.15). Therefore, differentiating $\lambda$ in Lemma 4.9 with respect to $x^{i}$ $(i=1,2)$, we have either $\sigma\left(\dot{x}^{1}, x^{2}\right)=\sigma\left(x^{1}, \dot{x}^{2}\right)=0$ or $u \dot{u}+v \dot{v}=0$. This implies $\sigma\left(\dot{x}^{1}, x^{2}\right)=\sigma\left(x^{1}, \dot{x}^{2}\right)=0$, because $\nu(t)$ is not constant. Thus, if $\mu \equiv 0$, then $\sigma\left(x^{1}, x^{2}\right)$ is constant.

Next, we assume $\left.A\left(x^{1}, \dot{x}^{2}\right)\right|_{H_{0}} \neq 0$. Then, we shall show that there is no curve $(u(t), v(t))$ satisfying the assumption for $\nu$ if $\sigma\left(x^{1}, x^{2}\right)$ is not constant. By the assumption $\left.A\left(x^{1}, \dot{x}^{2}\right)\right|_{H_{0}} \neq 0$, we have $\Phi\left(x^{1}, x^{2}, t\right)=A\left(x^{1}, x^{2}\right) P(t)$ in the same way to get Proposition 4.3. Furthermore, since we have Lemmas 4.3, 4.8 and 4.9 and Proposition 4.3 by $\left.A\left(\dot{x}^{1}, x^{2}\right)\right|_{H_{0}} \neq 0$, we may obtain similar results by $\left.A\left(x^{1}, \dot{x}^{2}\right)\right|_{H_{0}} \neq 0$ : We define functions $\bar{s}\left(x^{1}, x^{2}\right)$ and $\bar{\sigma}\left(x^{1}, x^{2}\right)$ by

$$
\left\{\begin{array}{l}
\bar{s}^{2}\left(x^{1}, x^{2}\right)=\left[\left(e^{h} h_{3}\right)^{2}+\left(e^{h} \mu\right)^{2}\right]\left(x^{1}, x^{2}, t\right), \\
\left(e^{h}\right)_{3}\left(x^{1}, x^{2}, t\right)=\bar{s}\left(x^{1}, x^{2}\right) \cos \left[\int_{t_{0}}^{t} \nu(t) d t+\bar{\sigma}\left(x^{1}, x^{2}\right)\right], \\
\left(\mu e^{h}\right)\left(x^{1}, x^{2}, t\right)=\bar{s}\left(x^{1}, x^{2}\right) \sin \left[\int_{t_{0}}^{t} \nu(t) d t+\bar{\sigma}\left(x^{1}, x^{2}\right)\right] .
\end{array}\right.
$$

Then, in the same way to get Lemma $4.9, \mu$ is represented as

$$
\mu\left(x^{1}, x^{2}, t\right)=\frac{\dot{v}(t) \sin \bar{\sigma}\left(x^{1}, x^{2}\right)-\dot{u}(t) \cos \bar{\sigma}\left(x^{1}, x^{2}\right)}{u(t) \sin \bar{\sigma}\left(x^{1}, x^{2}\right)+v(t) \cos \bar{\sigma}\left(x^{1}, x^{2}\right)} .
$$


We note $\bar{\sigma}\left(x^{1}, x^{2}\right) \neq \sigma\left(x^{1}, x^{2}\right)$. Indeed, if $\bar{\sigma}\left(x^{1}, x^{2}\right)=\sigma\left(x^{1}, x^{2}\right)$, then $\lambda\left(x^{1}, x^{2}, t\right)=$ $\mu\left(x^{1}, x^{2}, t\right)$, which condradicts the assumption. For each $\left(x^{1}, x^{2}\right)$, there exists $t$ such that $\mu\left(x^{1}, x^{2}, t\right) \neq 0$. Since $\lambda=\lambda\left(\sigma\left(x^{1}, x^{2}\right), t\right)$ and $\mu=\mu\left(\bar{\sigma}\left(x^{1}, x^{2}\right), t\right)$, there exists a function $F$ such that $\bar{\sigma}\left(x^{1}, x^{2}\right)=F\left(\sigma\left(x^{1}, x^{2}\right)\right)$ by (4.15). From (4.15), we obtain

$(4.15)^{\prime} q(t)=\frac{Y^{2} \sin \sigma \sin F(\sigma)+X^{2} \cos \sigma \cos F(\sigma)-X Y \sin (\sigma+F(\sigma))}{u^{2} \sin \sigma \sin F(\sigma)+v^{2} \cos \sigma \cos F(\sigma)+u v \sin (\sigma+F(\sigma))}$ where $X=\dot{u}+\nu v$ and $Y=\dot{v}-\nu u$. We assume that $\sigma$ is not constant. We differentiate $q(t)$ with respect to $\sigma$. From $u \dot{u}+v \dot{v} \not \equiv 0$, we have

$$
F_{\sigma}(\sigma)=-\frac{u Y \sin ^{2} F(\sigma)+\{-u X+v Y\} \sin F(\sigma) \cos F(\sigma)-v X \cos ^{2} F(\sigma)}{u Y \sin ^{2} \sigma+\{-u X+v Y\} \sin \sigma \cos \sigma-v X \cos ^{2} \sigma}
$$

Since $F$ is independent of $t$, we have $d\left(F_{\sigma}\right) / d t \equiv 0$. Putting $a(t)=(u Y)(t)$, $b(t)=(-u X+v Y)(t)$ and $c(t)=-(v X)(t)$, then we have

$\sin (F-\sigma)\{(\dot{a} b-a \dot{b}) \sin F \sin \sigma+(\dot{a} c-a \dot{c}) \sin (F+\sigma)+(\dot{b} c-b \dot{c}) \cos F \cos \sigma\}=0$.

Since $F(\sigma) \neq \sigma$, we have two cases:

(1) There exist constants $C_{1}$ and $C_{2}$ such that

$$
\begin{aligned}
& \left(\frac{b}{a}\right)(t)=\left(\frac{-u X+v Y}{u Y}\right)(t)=C_{1} \quad \text { and } \\
& \left(\frac{b}{c}\right)(t)=\left(\frac{-u X+v Y}{v X}\right)(t)=C_{2} .
\end{aligned}
$$

(2) There exist constants $C_{1}, C_{2}, C_{3}$ and a funciton $\ell(t)$ such that $\left(C_{1}, C_{2}, C_{3}\right) \neq(0,0,0)$ and

$$
\begin{aligned}
& C_{1} \sin F \sin \sigma+C_{2} \sin (F+\sigma)+C_{3} \cos F \cos \sigma=0, \\
& (\dot{a} b-a \dot{b})(t)=\ell(t) C_{1}, \quad(\dot{a} c-a \dot{c})(t)=\ell(t) C_{2}, \quad(\dot{b} c-b \dot{c})(t)=\ell(t) C_{3} .
\end{aligned}
$$

In the case (1), we have

$$
\frac{\dot{u}+\nu v}{\dot{v}-\nu u}=-C_{1}+\frac{v}{u}=\left(C_{2}+\frac{u}{v}\right)^{-1} .
$$

Therefore, the curve $(u(t), v(t))$ should be a quadratic curve (except circles). However, there is not any quadratic curve satifsying $(\dot{u}+\nu v) /(\dot{v}-\nu u)=$ 
$-C_{1}+v / u$. Thus, the case (1) does not happen. Furthermore, under our assumption for $\nu$, there is no curve satisfying the case (2). However, since this proof is too long, we shall prove this fact later, and continue the proof of Lemma.

Since there is no curve $(u(t), v(t))$ under the assumption that $\sigma$ is not constant, $\sigma\left(x^{1}, x^{2}\right)$ is constant function if $\left.A\left(x^{1}, \dot{x}^{2}\right)\right|_{H_{0}} \neq 0$.

If $\sigma\left(x^{1}, x^{2}\right)$ is constant, then $\lambda\left(x^{1}, x^{2}, t\right)=\lambda(t)$ and $\mu\left(x^{1}, x^{2}, t\right)=\mu(t)$ as we see before. If there exists a point $\left(a^{1}, a^{2}\right)$ such that $\left.A\left(a^{1}, \dot{a}^{2}\right)\right|_{H_{0}}=0$, then $\mu\left(a^{1}, a^{2}, t\right)=0$ for any $t$ from the proof of Proposition 4.2 and the assumption for $\nu$. Therefore, if there exists $\left(a^{1}, a^{2}\right)$ such that $\left.A\left(a^{1}, \dot{a}^{2}\right)\right|_{H_{0}} \neq 0$, then $\left.A\left(x^{1}, \dot{x}^{2}\right)\right|_{H_{0}} \neq 0$ for any $\left(x^{1}, x^{2}, t\right) \in U$ by the continuity of $\mu$, and $\sigma\left(x^{1}, x^{2}\right)$ is constant on $U$. Conversely, if there exists a point $\left(a^{1}, a^{2}\right)$ such that $\left.A\left(a^{1}, \dot{a}^{2}\right)\right|_{H_{0}}=0$, then $\left.A\left(x^{1}, \dot{x}^{2}\right)\right|_{H_{0}}=0$ for any $\left(x^{1}, x^{2}, t\right) \in U$ and $\mu \equiv 0$ on $U$. This completes the proof of Lemma.

Finally, we prove that there is no curve $(u(t), v(t))$ satisfying the case (2). The conditions on curve $(u(t), v(t))$ are as follows:

$$
\left\{\begin{array}{l}
(\dot{u} v-u \dot{v}) Y^{2}+u^{2}(\dot{X} Y-X \dot{Y})=\ell C_{1} \\
(\dot{u} v-u \dot{v}) X Y-u v(\dot{X} Y-X \dot{Y})=-\ell C_{2} \\
(\dot{u} v-u \dot{v}) X^{2}+v^{2}(\dot{X} Y-X \dot{Y})=\ell C_{3}
\end{array}\right.
$$

We note $\dot{X} Y-X \dot{Y}=\dot{\nu}(u \dot{u}+v \dot{v})$. Since $X^{2} Y^{2}=(X Y)^{2}$ and (4.17), we have

$$
\ell\left[C_{1} C_{3}-C_{2}^{2}\right]=\dot{\nu}\left(C_{1} v^{2}+C_{3} u^{2}-2 C_{2} u v\right)(u \dot{u}+v \dot{v})
$$

If $C_{1} C_{3}-C_{2}^{2}=0$, then the matrix $\left[\begin{array}{rr}C_{3} & -C_{2} \\ -C_{2} & C_{1}\end{array}\right]$ has zero-eigenvalue. Furthermore, $(u(t), v(t))$ for each $t$ is an eigenvector corresponding to the eigenvalue by $C_{1} v^{2}+C_{3} u^{2}-2 C_{2} u v=0$, that is, the curve $(u(t), v(t))$ is a line. Therefore, we may assume $C_{1} C_{3}-C_{2}^{2} \neq 0$.

From (4.17), we have

$$
\left\{\begin{array}{l}
(\dot{u} v-u \dot{v})\left(C_{2} Y^{2}+C_{1} X Y\right)+(\dot{X} Y-X \dot{Y})\left(C_{2} u^{2}-C_{1} u v\right)=0 \\
(\dot{u} v-u \dot{v})\left(C_{3} Y^{2}-C_{1} X^{2}\right)+(\dot{X} Y-X \dot{Y})\left(C_{3} u^{2}-C_{1} v^{2}\right)=0
\end{array}\right.
$$

Therefore, we have

$$
\left|\begin{array}{cc}
C_{2} Y^{2}+C_{1} X Y & C_{2} u^{2}-C_{1} u v \\
C_{3} Y^{2}-C_{1} X^{2} & C_{3} u^{2}-C_{1} v^{2}
\end{array}\right|=0
$$


Thus, there exists a function $Q(t)$ such that

$$
\left\{\begin{array}{l}
C_{2} Y^{2}+C_{1} X Y=Q\left(C_{3} Y^{2}-C_{1} X^{2}\right) \\
C_{2} u^{2}-C_{1} u v=Q\left(C_{3} u^{2}-C_{1} v^{2}\right)
\end{array}\right.
$$

When we consider coefficients of $\left(C_{2}-C_{3} Q\right)$ and $C_{1}$, we have

$$
\left|\begin{array}{cc}
Y^{2} & X Y+Q X^{2} \\
u^{2} & -u v+Q v^{2}
\end{array}\right|=0, \text { i.e., } Q=\frac{u Y}{v Y-u X} .
$$

From (4.20) and (4.21), we have

$$
\left(C_{2} v-C_{3} u\right) Y=\left(C_{2} u-C_{1} v\right) X .
$$

Therefore, there exists a function $R(t)$ such that

$$
X=R\left(C_{2} v-C_{3} u\right), \quad Y=R\left(C_{2} u-C_{1} v\right)
$$

Since

$$
\left\{\begin{array}{l}
\dot{u}+\nu v=X=-C_{3}(R u)+C_{2}(R v) \\
\dot{v}-\nu u=Y=C_{2}(R u)-C_{1}(R v)
\end{array}\right.
$$

we have

$$
\begin{aligned}
& u \dot{u}+v \dot{v}=-R\left(C_{1} v^{2}+C_{3} u^{2}-2 C_{2} u v\right), \\
& \left\{\begin{array}{l}
\frac{d(R u)}{d t}=\dot{R} u+R \dot{u}=\frac{\dot{\nu}\left(C_{2} u-C_{1} v\right)}{\left(C_{1} C_{3}-C_{2}^{2}\right)}, \\
\frac{d(R v)}{d t}=\dot{R} v+R \dot{v}=\frac{\dot{\nu}\left(C_{3} u-C_{2} v\right)}{\left(C_{1} C_{3}-C_{2}^{2}\right)} .
\end{array}\right.
\end{aligned}
$$

From (4.22) and (4.25), we have

$$
\dot{R}(u X+v Y)+R(\dot{u} X+\dot{v} Y)=0 .
$$

Next, we find the function $R(t)$ explicitly. From (4.18) and (4.24), we have $R=\dot{\nu}(u \dot{u}+v \dot{v})^{2} / \ell\left(C_{2}^{2}-C_{1} C_{3}\right)$. On the other hand, from (4.19) and (4.23), we have $R^{2}=\dot{\nu}(u \dot{u}+v \dot{v}) /\left\{(\dot{u} v-u \dot{v})\left(C_{1} C_{3}-C_{2}^{2}\right)\right\}$. Thus, we have $R^{2}=-\ell R /\{(\dot{u} v+u \dot{v})(\dot{u} v-u \dot{v})\}$. Therefore, we have another expression for $R(t)$ :

$$
R(t)=-\ell\{(u \dot{u}+v \dot{v})(\dot{u} v-u \dot{v})\}^{-1}
$$


Since

$$
\frac{d(u \dot{u}+v \dot{v})}{d t}=1+\nu(\dot{u} v-u \dot{v}), \quad \frac{d(\dot{u} v-u \dot{v})}{d t}=-\nu(u \dot{u}+v \dot{v})
$$

we have $\dot{\ell} / \ell=-\nu(u \dot{u}+v \dot{v}) /(\dot{u} v-u \dot{v})$ by $(4.26),(4.27)$ and $\dot{u}^{2}+\dot{v}^{2}=1$. If $\dot{\ell}(t) \equiv 0$, then $u \dot{u}+v \dot{v} \equiv 0$, which contradicts the assumption for $\nu$. Therefore, we may take $\ell(t)=(\dot{u} v-u \dot{v})(t)$. Then, we have $R(t)=-(u \dot{u}+$ $v \dot{v})^{-1}(t)$ by $(4.27)$, and $\dot{\nu}=\left(C_{1} C_{3}-C_{2}^{2}\right)(\dot{u} v-u \dot{v}) /(u \dot{u}+v \dot{v})^{3}$. Furthermore, we have

$$
\left\{\begin{array}{l}
Y^{2}+\left(C_{1} C_{3}-C_{2}^{2}\right)\left(\frac{u}{u \dot{u}+v \dot{v}}\right)^{2}=C_{1}, \\
X^{2}+\left(C_{1} C_{3}-C_{2}^{2}\right)\left(\frac{v}{u \dot{u}+v \dot{v}}\right)^{2}=C_{3}, \\
X Y-\left(C_{1} C_{3}-C_{2}^{2}\right) \frac{u v}{(u \dot{u}+v \dot{v})^{2}}=-C_{2}
\end{array}\right.
$$

by (4.17). We have to consider two cases $C_{1} C_{3}-C_{2}^{2}>0$ and $C_{2}^{2}-C_{1} C_{3}>0$ in $(4.17)^{\prime}$.

Assume $C_{1} C_{3}-C_{2}^{2}>0, C_{1}>0$ and $C_{3}>0$. Then, there exists a function $\varphi(t)$ such that

$$
\left\{\begin{array}{l}
\left(Y, \sqrt{C_{1} C_{3}-C_{2}^{2}} u /(u \dot{u}+v \dot{v})\right)=\left(\sqrt{C_{1}} \cos \varphi, \sqrt{C_{1}} \sin \varphi\right) \\
\left(X,-\sqrt{C_{1} C_{3}-C_{2}^{2}} v /(u \dot{u}+v \dot{v})\right) \\
\quad=\left(\sqrt{C_{3}} \cos (\varphi+C), \sqrt{C_{3}} \sin (\varphi+C)\right)
\end{array}\right.
$$

by $(4.17)^{\prime}$, where $\sqrt{C_{1} C_{3}} \cos C=-C_{2}$. On the other hand, if (4.28) holds, then we have $C_{2}^{2}=C_{1} C_{3}$ or $C_{2}=0$. Indeed, since we have

$$
C_{1} C_{3}-C_{2}^{2}=C_{1} C_{3}\left[\sin ^{2}(\varphi+C)+\sin ^{2} \varphi\right]+2 C_{2} \sqrt{C_{1} C_{3}} \sin \varphi \sin (\varphi+C)
$$

by (4.24) and (4.28), we have

$$
C_{1} C_{3}[\sin (\varphi+C) \cos (\varphi+C)+\sin \varphi \cos \varphi]+C_{2} \sqrt{C_{1} C_{3}} \sin (2 \varphi+C)=0
$$

by differentiating the equation and $\dot{\varphi}(t) \not \equiv 0$. Thus, we have $\cos C=0$ or $\cos C= \pm 1$. We have only to consider the case $C_{2}=0$. By the condition of the case (2) and $C_{2}=0$, we have $\tan \sigma \tan F=-C_{3} / C_{1}$. Furthermore, we have that $(\tan \sigma+\tan F)$ is constant. Indeed, if $\tan \sigma \tan F$ is constant 
but $(\tan \sigma+\tan F)$ is not constant, then $q(t)$ has to depend on $\sigma$ by $(4.15)^{\prime}$ and $C_{2}=0$. Thus, both functions $\tan \sigma$ and $\tan F(\sigma)$ have to be constant. However, since we consider the case (2) under the condition that $\sigma\left(x^{1}, x^{2}\right)$ is not constant, this case does not occur.

Next, we assume $C_{2}^{2}-C_{1} C_{3}>0$. First, we argue the case $C_{1}>0$ and $C_{3}>0$. We may consider the case $C_{1}<0$ and $C_{3}<0$ in the same way as the case $C_{1}>0$ and $C_{3}>0$. We may take a function $\varphi(t)$ such that

$$
\left\{\begin{array}{l}
\left(Y, \sqrt{C_{2}^{2}-C_{1} C_{3}} u /(u \dot{u}+v \dot{v})\right)=\left(\sqrt{C_{1}} \cosh \varphi, \sqrt{C_{1}} \sinh \varphi\right), \\
\left(X, \sqrt{C_{2}^{2}-C_{1} C_{3}} v /(u \dot{u}+v \dot{v})\right)=\left(\sqrt{C_{3}} \cosh (C-\varphi), \sqrt{C_{3}} \sinh (C-\varphi)\right),
\end{array}\right.
$$

where $\cosh C=-C_{2} / \sqrt{C_{1} C_{3}}(>1)$. However, we have $C=0$ and $\sqrt{C_{1} C_{3}}=$ $-C_{2}$ in the same way as the case $C_{1} C_{3}-C_{2}^{2}>0$, which contradicts $C_{2}^{2}-$ $C_{1} C_{3}>0$.

If $C_{2}^{2}-C_{1} C_{3}>0, C_{1}>0$ and $C_{3}<0$, then we have $C_{2}=0$ in the same way as above. However, this case does not occur as we see before.

Therefore, there is no curve satisfying both conditions of the case (2) and assumption for $\nu$.

This completes the proof of Proposition 4.4.

Proposition 4.5. If there exists a point $\left(a^{1}, a^{2}\right)$ such that $\left.A\left(\dot{a}^{1}, a^{2}\right)\right|_{H_{0}}$ $\neq 0$, then $\left.A\left(\dot{x}^{1}, x^{2}\right)\right|_{H_{0}} \neq 0$ for any $\left(x^{1}, x^{2}\right)$.

Proof. Assume that there exists a point $\left(x^{1}, x^{2}\right)$ such that $\left.A\left(\dot{x}^{1}, x^{2}\right)\right|_{H_{0}}$ $=0$. Let us take a point $p=\left(b^{1}, b^{2}, t_{0}\right) \in(\bar{U} \backslash U) \cap M$. Then, we have $\left.A\left(\dot{b}^{1}, b^{2}\right)\right|_{H_{0}}=0$ by the definition of $U$. Then $(\lambda \nu)\left(b^{1}, b^{2}, t\right)=0$ for any $t$ from the proof of Proposition 4.2. From $p=\left(b^{1}, b^{2}, t_{0}\right) \in(\bar{U} \backslash U)$ and $\lambda\left(x^{1}, x^{2}, t\right)=\lambda(t)$ on $U$, we have $\lambda\left(b^{1}, b^{2}, t\right)=\lambda(t)$ for any $t$ and $(\lambda \nu)(t)=0$, and then $\lambda(t)=0$ for any $\left(x^{1}, x^{2}, t\right) \in U$ by the assumption for $\nu$, which contradicts the definition of $U$ by Lemma 4.9-(2).

The conclusions of Lemma 4.3 and Propositions 4.3 and 4.4 hold true on $M$ by Proposition 4.5. From now on, we start our discussion on whole $M$. We prove at first Theorem 2-(3a).

Proposition 4.6. We assume that there exists a point $\left(a^{1}, a^{2}\right)$ such that $\left.A\left(\dot{a}^{1}, a^{2}\right)\right|_{H_{0}} \neq 0$ and $\left.A\left(a^{1}, \dot{a}^{2}\right)\right|_{H_{0}}=0$. Then, we have $\lambda\left(x^{1}, x^{2}, t\right)=$ $\lambda(t), \mu \equiv 0$. Furthermore, the metric $g$ is given by $g=g_{S}+\left(d x^{2}\right)^{2}$, where $g_{S}$ is the metric of a surface $S$ of revolution in Euclidean 3 -space $\mathbf{R}^{3}$ with constant Gaussian curvature. 
Proof. We have $\left.A\left(\dot{x}^{1}, x^{2}\right)\right|_{H_{0}} \neq 0$ and $\left.A\left(x^{1}, \dot{x}^{2}\right)\right|_{H_{0}}=0$ for any $\left(x^{1}, x^{2}\right)$ by Proposition 4.5. Then, we have $\lambda=\lambda(t)$ by Proposition 4.4, and $\mu \equiv 0$, $\lambda(t) \neq 0$ and $\nu(t) \neq 0$ from the proof of Proposition 4.2 and assumptions of genericalness and for $\nu$. Furthermore, each $x^{2}$-curve is a part of line in $\mathbf{R}^{4}$ by $\mu \equiv 0$ and (2.3). In this case, the equation (4.12) becomes as follows:

$$
M: \Phi\left(x^{1}, x^{2}, t\right)=A\left(x^{1}\right) P(t)+\mathbf{a} x^{2},
$$

where $A\left(x^{1}\right) \in S O(4)$ and $\mathbf{a} \in \mathbf{R}^{4}$, because of $\mathbf{a}\left(x^{1}, x^{2}\right)=\mathbf{a}\left(x^{2}\right)$ by Proposition 4.3. Furthermore, we have

$$
\nabla_{X_{\alpha}}^{\prime 2} X_{\alpha}=-\left[\left(\frac{\lambda_{3}}{\lambda-\nu}\right)^{2}+\lambda^{2}\right] X_{\alpha}
$$

by (2.3). Since the coefficient of $X_{\alpha}$ in $\nabla_{X_{\alpha}}^{\prime 2} X_{\alpha}$ depends only on $t$, each $x^{1}$-curve is a circle in $\mathbf{R}^{4}$. Therefore, we may assume

$$
A\left(x^{1}\right) P(t)=\left[\begin{array}{ccc}
\cos x^{1} & -\sin x^{1} & 0 \\
\sin x^{1} & \cos x^{1} & \\
0 & & I
\end{array}\right]\left[\begin{array}{c}
u(t) \\
0 \\
v(t) \\
0
\end{array}\right], \quad \mathbf{a}=\left[\begin{array}{l}
0 \\
0 \\
0 \\
1
\end{array}\right],
$$

because $X_{\beta}$ is perpendicular to the plane $H_{0}$ and $X_{\alpha}$, and any $\left(x^{1}, t\right)$-surface is not included in a plane. Furthermore, the Gaussian curvature $K$ of $\left(x^{1}, t\right)$ surface is given by $K=\lambda \nu$, and then $\lambda \nu$ is constant by Theorem 3-(2).

We continue the proof of Theorem 2-(3a). The assumption $\left.A\left(a^{1}, \dot{a}^{2}\right)\right|_{H_{0}}$ $=0$ in Proposition 4.6 can be replaced by $h_{3}\left(a^{1}, a^{2}, t\right)=0$. Indeed, if $\left.A\left(a^{1}, \dot{a}^{2}\right)\right|_{H_{0}}=0$, then $h_{3}\left(a^{1}, a^{2}, t\right)=0$ from the proof of Proposition 4.2. Conversely, if $h_{3}\left(a^{1}, a^{2}, t\right)=0$, then $0=\left(-h_{33}-h_{3}^{2}\right)\left(a^{1}, a^{2}, t\right)=\mu \nu\left(a^{1}, a^{2}, t\right)$. Thus, we have $\mu\left(a^{1}, a^{2}, t\right)=0$ by the assumption for $\nu$. Furthermore, since $\mu=\mu(t)$ by Proposition 4.4 and $\left.A\left(\dot{a}^{1}, a^{2}\right)\right|_{H_{0}} \neq 0$, we have $\mu \equiv 0$. This shows that each $x^{2}$-curve is a line and $\left.A\left(x^{1}, \dot{x}^{2}\right)\right|_{H_{0}}=0$ for any $\left(x^{1}, x^{2}\right)$ in the same way to get $(4.12)^{\prime}$ in proof of Proposition 4.6. This fact together with Proposition 4.6 completes the proof of Theorem 2-(3a).

We still leave the proof of Theorem 2-(3b). We start its proof. Assume that there exists a point $\left(a^{1}, a^{2}\right)$ such that $\left.A\left(\dot{a}^{1}, a^{2}\right)\right|_{H_{0}} \neq 0$ and $\left.A\left(a^{1}, \dot{a}^{2}\right)\right|_{H_{0}} \neq 0$. Then, we have

(1) $\left.A\left(\dot{x}^{1}, x^{2}\right)\right|_{H_{0}} \neq 0$ and $\left.A\left(x^{1}, \dot{x}^{2}\right)\right|_{H_{0}} \neq 0$ for any $\left(x^{1}, x^{2}\right)$,

(2) $\lambda\left(x^{1}, x^{2}, t\right)=\lambda(t)$ and $\mu\left(x^{1}, x^{2}, t\right)=\mu(t)$, 
(3) $P(t)=u(t) \boldsymbol{b}+v(t) \boldsymbol{c}$, where $A\left(\dot{x}^{1}, x^{2}\right) \boldsymbol{b}=0$ and $A\left(\dot{x}^{1}, x^{2}\right) \boldsymbol{c}=$ $s\left(x^{1}, x^{2}\right) X_{\alpha}\left(x^{1}, x^{2}\right)$, and $\boldsymbol{b}$ and $\boldsymbol{c}$ do not depend on $\left(x^{1}, x^{2}\right)$.

In the same way to get (1), (2) and (3) from $\left.A\left(\dot{a}^{1}, a^{2}\right)\right|_{H_{0}} \neq 0$, we also define frames $\{\overline{\boldsymbol{b}}, \overline{\boldsymbol{c}}\}$ of $H_{0}$ and a positive function $\bar{s}\left(x^{1}, x^{2}\right)$ from $\left.A\left(a^{1}, \dot{a}^{2}\right)\right|_{H_{0}} \neq 0$ by

$$
\left\{\begin{array}{l}
\overline{\boldsymbol{b}}\left(x^{1}, x^{2}, t\right)=\frac{-\mu \boldsymbol{e}_{1}-h_{3} \boldsymbol{e}_{2}}{\left[h_{3}^{2}+\mu^{2}\right]^{1 / 2}}\left(x^{1}, x^{2}, t\right) \\
\overline{\boldsymbol{c}}\left(x^{1}, x^{2}, t\right)=\frac{h_{3} \boldsymbol{e}_{1}-\mu \boldsymbol{e}_{2}}{\left[h_{3}^{2}+\mu^{2}\right]^{1 / 2}}\left(x^{1}, x^{2}, t\right) \\
\bar{s}^{2}\left(x^{1}, x^{2}, t\right)=\left[\left(e^{h} h_{3}\right)^{2}+\left(e^{h} \mu\right)^{2}\right]\left(x^{1}, x^{2}, t\right) .
\end{array}\right.
$$

We have the following:

(4) Both vectors $\overline{\boldsymbol{b}}$ and $\overline{\boldsymbol{c}}$ are constant, and $\bar{s}\left(x^{1}, x^{2}, t\right)=\bar{s}\left(x^{1}, x^{2}\right)$.

(5) $A\left(x^{1}, \dot{x}^{2}\right) \overline{\boldsymbol{b}}=0$ and $A\left(x^{1}, \dot{x}^{2}\right) \overline{\boldsymbol{c}}=\bar{s}\left(x^{1}, x^{2}\right) X_{\beta}\left(x^{1}, x^{2}\right)$.

(6) Putting $P(t)=\bar{u}(t) \overline{\boldsymbol{b}}+\bar{v}(t) \overline{\boldsymbol{c}}$, then there exists a constant $\tau$ such that

$$
\left\{\begin{array}{l}
\dot{\bar{u}}(t)=-\sin \left[\int_{t_{0}}^{t} \nu(t) d t+\tau\right], \quad \dot{\bar{v}}(t)=\cos \left[\int_{t_{0}}^{t} \nu(t) d t+\tau\right], \\
\bar{v}(t)=\frac{1}{\mu} \sin \left[\int_{t_{0}}^{t} \nu(t) d t+\tau\right], \\
\left(e^{h}\right)_{3}\left(x^{1}, x^{2}, t\right)=\bar{s}\left(x^{1}, x^{2}\right) \cos \left[\int_{t_{0}}^{t} \nu(t) d t+\tau\right] .
\end{array}\right.
$$

(7) Putting $\tau=\sigma+\theta$, then $\theta \neq 0$.

(8) $\Phi\left(x^{1}, x^{2}, t\right)=A\left(x^{1}, x^{2}\right) P(t)$, i.e., $\mathbf{a}\left(x^{1}, x^{2}\right)=\mathbf{0}$ in (4.12).

Proposition 4.7. We assume that there exists a point $\left(a^{1}, a^{2}\right)$ such that $\left.A\left(\dot{a}^{1}, a^{2}\right)\right|_{H_{0}} \neq 0$ and $\left.A\left(a^{1}, \dot{a}^{2}\right)\right|_{H_{0}} \neq 0$. Then we have the following:

(1) By choosing a suitable admissible coordinate system if necessary, we may assume that both functions $f\left(x^{1}, x^{2}, t\right)$ and $h\left(x^{1}, x^{2}, t\right)$ depend only on variable $t$, i.e., $f\left(x^{1}, x^{2}, t\right)=f(t)$ and $h\left(x^{1}, x^{2}, t\right)=h(t)$, and $s\left(x^{1}, x^{2}\right)=$ $\bar{s}\left(x^{1}, x^{2}\right)=1$.

(2) $\theta= \pm \pi / 2$.

(3) $\Phi\left(x^{1}, x^{2}, t\right)=\left[\begin{array}{cc}A_{2}\left(x^{2}\right) & 0 \\ 0 & A_{1}\left(x^{1}\right)\end{array}\right]\left[\begin{array}{c}u(t) \\ 0 \\ v(t) \\ 0\end{array}\right]$, where the plane curve $(u(t), v(t))$ satisfies $(\dot{u}(t))^{2}+(\dot{v}(t))^{2}=1$ and $(\ddot{u}(t), \ddot{v}(t))=\nu(t)(-\dot{v}(t), \dot{u}(t))$. 
Proof. (1) Since $f_{3}=-\lambda_{3} /(\lambda-\nu), h_{3}=-\mu_{3} /(\mu-\nu), \lambda=\lambda(t)$, $\mu=\mu(t)$ and $\nu=\nu(t)$, functions $f_{3}$ and $h_{3}$ depend only on variable $t$. Since $R_{213}^{1}=0$ and $f_{23}=0$, we have $f_{2}\left(f_{3}-h_{3}\right)=0$. Furthermore, since $R_{223}^{1}=0$ and $h_{13}=0$, we have $h_{1}\left(h_{3}-f_{3}\right)=0$. Thus we have either $f_{2}=h_{1}=0$ or $f_{3}=h_{3}$. If $f_{3}=h_{3}$, then $\lambda=\mu$ by the Gauss equation and the assumption for $\nu$. Therefore we have $f_{2}=h_{1}=0$. Moreover, since $f_{13}=h_{23}=0$, we have $f\left(x^{1}, t\right)=\bar{f}\left(x^{1}\right)+\overline{\bar{f}}(t)$ and $h\left(x^{2}, t\right)=\bar{h}\left(x^{2}\right)+\overline{\bar{h}}(t)$. Thus we may assume $f\left(x^{1}, x^{2}, t\right)=f(t)$ and $h\left(x^{1}, x^{2}, t\right)=h(t)$ by changing the admissible coordinate system. Then, since $\left(e^{f}\right)_{3}=s\left(x^{1}, x^{2}\right) \cos \left[\int_{t_{0}}^{t} \nu(t) d t+\sigma\right]$ and $\left(e^{h}\right)_{3}=\bar{s}\left(x^{1}, x^{2}\right) \cos \left[\int_{t_{0}}^{t} \nu(t) d t+\tau\right], s$ and $\bar{s}$ are constant. In particular, we may take $s=\bar{s}=1$.

(2) and (3). Since $\nabla_{X_{\alpha}}^{\prime 2} X_{\alpha}=-\left[\left(\frac{\lambda_{3}}{\lambda-\nu}\right)^{2}+\lambda^{2}\right] X_{\alpha}$ and $\nabla_{X_{\beta}}^{\prime 2} X_{\beta}=-\left[\left(\frac{\mu_{3}}{\mu-\nu}\right)^{2}+\right.$ $\left.\mu^{2}\right] X_{\beta}$, each $x^{i}$-curve $(i=1,2)$ is a part of circle in $\mathbf{R}^{4}$. Since each $x^{1}$-curve $A\left(x^{1}, x^{2}\right) \boldsymbol{c}$ for fixed $x^{2}$ is a unit circle and $A\left(\dot{x}^{1}, x^{2}\right) \boldsymbol{c}=X_{\alpha}\left(x^{1}, x^{2}\right)$, we have $\left(A^{-1}\left(x^{1}, x^{2}\right) A\left(\dot{x}^{1}, x^{2}\right)\right) \boldsymbol{c}=A^{-1}\left(x^{1}, x^{2}\right) X_{\alpha}\left(x^{1}, x^{2}\right)=X_{\alpha}\left(0, x^{2}\right)$. Furthermore, since $\nabla_{X_{\beta}}^{\prime} X_{\alpha}=\frac{-\mu_{\alpha}}{\mu-\lambda} X_{\beta}=0$, we have $\left(A^{-1}\left(x^{1}, x^{2}\right)\right.$ $\left.A\left(\dot{x}^{1}, x^{2}\right)\right) \boldsymbol{c}=X_{\alpha}(0,0)$. We put $B=\left(A^{-1}\left(x^{1}, x^{2}\right) A\left(\dot{x}^{1}, x^{2}\right)\right)$. The matrix $B$ is constant and skew-symmetric, and it satisfies

$$
B \boldsymbol{b}=\mathbf{0}, \quad B \boldsymbol{c}=X_{\alpha}(0,0), \quad B X_{\alpha}(0,0)=-\boldsymbol{c}, \quad B X_{\beta}(0,0)=\mathbf{0}
$$

We put $C=A^{-1}\left(x^{1}, x^{2}\right) A\left(x^{1}, \dot{x}^{2}\right)$. Similarly, since $\nabla_{X_{\alpha}}^{\prime} X_{\beta}=-\frac{\lambda_{\beta}}{\lambda-\mu} X_{\alpha}=0$, we have

$$
C \overline{\boldsymbol{b}}=\mathbf{0}, C \overline{\boldsymbol{c}}=X_{\beta}(0,0), C X_{\alpha}(0,0)=\mathbf{0}, C X_{\beta}(0,0)=-\overline{\boldsymbol{c}}
$$

Since $B_{2}=C_{1}=0$, we have

$$
B C=C B=A^{-1}\left(x^{1}, x^{2}\right) A\left(\dot{x}^{1}, \dot{x}^{2}\right)
$$

Since $C \boldsymbol{c}=-C B X_{\alpha}(0,0)=-B C X_{\alpha}(0,0)=\mathbf{0}$ by (4.29) and (4.30), we have $\boldsymbol{c}= \pm \overline{\boldsymbol{b}}$. Therefore, we have $\{\boldsymbol{b}, \boldsymbol{c}\}=\{ \pm \overline{\boldsymbol{c}}, \mp \overline{\boldsymbol{b}}\}$, because $\{\boldsymbol{b}, \boldsymbol{c}\}$ and $\{\overline{\boldsymbol{b}}, \overline{\boldsymbol{c}}\}$ are oriented orthonormal frames of $H_{0}$. Thus we have $\theta=$ $\pm \pi / 2$. In particular, we note $B C=C B=0$ by (4.29) and (4.30), i.e., $A\left(\dot{x}^{1}, \dot{x}^{2}\right)=0$ by (4.31). We assume $\theta=\pi / 2$, i.e., $\{\boldsymbol{b}, \boldsymbol{c}\}=\{\overline{\boldsymbol{c}},-\overline{\boldsymbol{b}}\}$. Since both $A\left(\dot{x}^{1}, x^{2}\right) \boldsymbol{c}=X_{\alpha}$ and $A\left(x^{1}, \dot{x}^{2}\right) \boldsymbol{b}=X_{\beta}$ are unit vectors, and each 
$x^{i}$-curve $(i=1,2)$ is a circle in $\mathbf{R}^{4}, A\left(\dot{x}^{1}, \dot{x}^{2}\right)=0$ and $\theta=\pi / 2$, we have

$$
\Phi\left(x^{1}, x^{2}, t\right)=\left[\begin{array}{cc}
A\left(x^{2}\right) & 0 \\
0 & A\left(x^{1}\right)
\end{array}\right]\left[\begin{array}{c}
u(t) \\
0 \\
v(t) \\
0
\end{array}\right] .
$$

Since $(u(t), v(t))$ has an arc-length parameter and curvature $\nu$, we have $(\dot{u}(t))^{2}+(\dot{v}(t))^{2}=1$ and $(\ddot{u}(t), \ddot{v}(t))=\nu(t)(-\dot{v}(t), \dot{u}(t))$.

For the convenient of the calculation, we denote $\dot{u}\left(=u_{3}\right)$ by $u^{\prime}$ for a function $u=u(t)$ from now on. Next, we prove the last equation of (1.1) in Theorem 2-(3b).

Proposition 4.8. The plane curve $(u(t), v(t))$ given in Proposition 4.7(3) satisfies the following condition: There exist positive constants a and $b$ such that $a^{2}\left(u^{\prime}+\nu v\right)^{2} \pm b^{2}\left(v^{\prime}-\nu u\right)^{2}=1$.

The proof of Proposition 4.8 is divided into Lemmas 4.11, 4.12, 4.13 and 4.14 below.

LEMma 4.11. The following equation holds:

$$
u v\left(u u^{\prime}+v v^{\prime}\right) \nu^{\prime}+\left(u v^{\prime}-u^{\prime} v\right)\left(u^{\prime}+\nu v\right)\left(v^{\prime}-\nu u\right)=0 .
$$

Proof. Since the unit vector field $N$ normal to the hypersurface $M$ : $\left(x^{1}, x^{2}, t\right) \mapsto \Phi\left(x^{1}, x^{2}, t\right) \in \mathbf{R}^{4}$ is given by

$$
N=\left[\begin{array}{cc}
A\left(x^{2}\right) & 0 \\
0 & A\left(x^{1}\right)
\end{array}\right]\left[\begin{array}{r}
-v^{\prime}(t) \\
u^{\prime}(t)
\end{array}\right]
$$

we have $\lambda=-u^{\prime} / v$ and $\mu=v^{\prime} / u$ by $X_{\alpha}=v^{-1} \partial / \partial x^{1}$ and $X_{\beta}=u^{-1} \partial / \partial x^{2}$, where we denote ${ }^{t}\left(-v^{\prime}(t), 0, u^{\prime}(t), 0\right)$ by ${ }^{t}\left(-v^{\prime}(t), u^{\prime}(t)\right)$ for the simplicity. The principal curvatures $\lambda(t), \mu(t)$ and $\nu(t)$ satisfy the equation

$$
(\lambda-\mu) \nu^{\prime}+(\nu-\mu) \lambda^{\prime}+(\lambda-\nu) \mu^{\prime}=0
$$

by Theorem $3-(2)$. Using the equations $\left(u^{\prime}\right)^{2}+\left(v^{\prime}\right)^{2}=1$ and $\left(u^{\prime \prime}, v^{\prime \prime}\right)=$ $\nu\left(-v^{\prime}, u^{\prime}\right)$, we have

$$
0=\left(-\frac{u^{\prime}}{v}-\frac{v^{\prime}}{u}\right) \nu^{\prime}+\left(\nu-\frac{v^{\prime}}{u}\right)\left(\frac{-u^{\prime \prime} v+u^{\prime} v^{\prime}}{v^{2}}\right)
$$




$$
\begin{aligned}
& +\left(-\frac{u^{\prime}}{v}-\nu\right)\left(\frac{v^{\prime \prime} u-u^{\prime} v^{\prime}}{u^{2}}\right) \\
= & \frac{-\nu^{\prime}}{u v}\left(u u^{\prime}+v v^{\prime}\right)+\frac{v^{\prime}}{u v^{2}}\left(\nu u-v^{\prime}\right)\left(\nu v+u^{\prime}\right)-\frac{u^{\prime}}{u^{2} v}\left(u^{\prime}+\nu v\right)\left(\nu u-v^{\prime}\right) \\
= & \frac{-\nu^{\prime}}{u v}\left(u u^{\prime}+v v^{\prime}\right)-\frac{1}{u^{2} v^{2}}\left(u v^{\prime}-u^{\prime} v\right)\left(u^{\prime}+\nu v\right)\left(v^{\prime}-\nu u\right) .
\end{aligned}
$$

LEMMA 4.12. The equation (4.33) is equivalent to each of the following equations (4.34): There exist constants $A$ and $B$ such that

$$
u=A\left(u^{\prime}+\nu v\right)\left(u u^{\prime}+v v^{\prime}\right), \quad v=B\left(v^{\prime}-\nu u\right)\left(u u^{\prime}+v v^{\prime}\right)
$$

Proof. Since

$$
\left\{\begin{array}{l}
\left(u u^{\prime}+v v^{\prime}\right)^{\prime}=\left(u^{\prime}\right)^{2}+\left(v^{\prime}\right)^{2}+u u^{\prime \prime}+v v^{\prime \prime}=1+\nu\left(-u v^{\prime}+u^{\prime} v\right) \\
\left(u^{\prime}+\nu v\right)^{\prime}=u^{\prime \prime}+\nu v^{\prime}+\nu^{\prime} v=\nu^{\prime} v \\
\left(v^{\prime}-\nu u\right)^{\prime}=v^{\prime \prime}-\nu u^{\prime}-\nu^{\prime} u=-\nu^{\prime} u
\end{array}\right.
$$

we have the following equation by (4.33):

$$
\begin{aligned}
& u v\left(u u^{\prime}+v v^{\prime}\right) \nu^{\prime}+\nu\left(u v^{\prime}-u^{\prime} v\right) v\left(v^{\prime}-\nu u\right)+u^{\prime}\left(u v^{\prime}-u^{\prime} v\right)\left(v^{\prime}-\nu u\right) \\
& =u v\left(u u^{\prime}+v v^{\prime}\right) \nu^{\prime}-\left[1+\nu\left(-u v^{\prime}+u^{\prime} v\right)\right] v\left(v^{\prime}-\nu u\right) \\
& \quad+\left[v+u u^{\prime} v^{\prime}-\left(u^{\prime}\right)^{2} v\right]\left(v^{\prime}-\nu u\right) \\
& =u v\left(u u^{\prime}+v v^{\prime}\right) \nu^{\prime}-\left[1+\nu\left(-u v^{\prime}+u^{\prime} v\right)\right] v\left(v^{\prime}-\nu u\right) \\
& \quad+\left(u u^{\prime}+v v^{\prime}\right) v^{\prime}\left(v^{\prime}-\nu u\right) \\
& =-v\left(u u^{\prime}+v v^{\prime}\right)\left(v^{\prime}-\nu u\right)^{\prime}-\left(u u^{\prime}+v v^{\prime}\right)^{\prime} v\left(v^{\prime}-\nu u\right) \\
& \quad+\left(u u^{\prime}+v v^{\prime}\right) v^{\prime}\left(v^{\prime}-\nu u\right) \\
& =0
\end{aligned}
$$

If $u u^{\prime}+v v^{\prime} \equiv 0$, then $(u(t), v(t))$ is a circle in $H_{0}$. Then $M$ is a sphere in $\mathbf{R}^{4}$, which is a contradiction to the assumption. Since $(u(t), v(t))$ is not a circle, we have $v^{\prime}-\nu u \not \equiv 0$ and

$$
-\frac{\left(v^{\prime}-\nu u\right)^{\prime}}{v^{\prime}-\nu u}-\frac{\left(u u^{\prime}+v v^{\prime}\right)^{\prime}}{u u^{\prime}+v v^{\prime}}+\frac{v^{\prime}}{v}=0 .
$$


This shows the second equation of (4.34). Similarly, we have, from (4.33),

$$
\begin{aligned}
& u v\left(u u^{\prime}+v v^{\prime}\right) \nu^{\prime}-\nu\left(u v^{\prime}-u^{\prime} v\right) u\left(u^{\prime}+\nu v\right)+v^{\prime}\left(u v^{\prime}-u^{\prime} v\right)\left(u^{\prime}+\nu v\right) \\
& =u v\left(u u^{\prime}+v v^{\prime}\right) \nu^{\prime}+\left[1+\nu\left(-u v^{\prime}+u^{\prime} v\right)\right] u\left(u^{\prime}+\nu v\right) \\
& \quad+\left[-u+u\left(v^{\prime}\right)^{2}-v u^{\prime} v^{\prime}\right]\left(u^{\prime}+\nu v\right) \\
& =u\left(u u^{\prime}+v v^{\prime}\right)\left(u^{\prime}+\nu v\right)^{\prime}+u\left(u^{\prime}+\nu v\right)\left(u u^{\prime}+v v^{\prime}\right)^{\prime} \\
& \quad-u^{\prime}\left(u u^{\prime}+v v^{\prime}\right)\left(u^{\prime}+\nu v\right) \\
& =0
\end{aligned}
$$

that is,

$$
\frac{\left(u^{\prime}+\nu v\right)^{\prime}}{u^{\prime}+\nu v}+\frac{\left(u u^{\prime}+v v^{\prime}\right)^{\prime}}{u u^{\prime}+v v^{\prime}}-\frac{u^{\prime}}{u}=0 .
$$

This shows the first equation of (4.34).

We have

$$
\frac{u}{v}=\frac{A}{B} \frac{u^{\prime}+\nu v}{v^{\prime}-\nu u}, \text { and then }-\frac{\left(-\nu^{\prime} u\right)}{\nu^{\prime} v}=\frac{A}{B} \frac{u^{\prime}+\nu v}{v^{\prime}-\nu u}
$$

by (4.34). Thus, we have

$$
A\left(u^{\prime}+\nu v\right)\left(u^{\prime}+\nu v\right)^{\prime}+B\left(v^{\prime}-\nu u\right)\left(v^{\prime}-\nu u\right)^{\prime}=0
$$

by (4.35). Therefore, there exists a constant $C$ such that

$$
A\left(u^{\prime}+\nu v\right)^{2}+B\left(v^{\prime}-\nu u\right)^{2}=C .
$$

Lemma 4.13. We have $C \neq 0$ in (4.36).

Proof. We assume $C=0$. Then, we may assume $A=a^{2}$ and $B=-b^{2}$. Thus we have

$$
\left[a\left(u^{\prime}+\nu v\right)+b\left(v^{\prime}-\nu u\right)\right]\left[a\left(u^{\prime}+\nu v\right)-b\left(v^{\prime}-\nu u\right)\right]=0,
$$

that is,

$$
a u^{\prime}+b v^{\prime}=-\nu(a v-b u) \quad \text { or } \quad a u^{\prime}-b v^{\prime}=-\nu(a v+b u)
$$

If $a u^{\prime}+b v^{\prime}=-\nu(a v-b u)$, then $a u^{\prime \prime}+b v^{\prime \prime}=-\nu\left(a v^{\prime}-b u^{\prime}\right)-\nu^{\prime}(a v-b u)$, that is, $\nu^{\prime}(a v-b u)=0$. If $\nu^{\prime} \equiv 0$, then $(u(t), v(t))$ is a circle. If $a v(t)-b u(t) \equiv 0$, then $(u(t), v(t))$ is a line, that is, $\nu \equiv 0$. Neither case happens. Similarly, the case $a u^{\prime}-b v^{\prime}=-\nu(a v+b u)$ also does not happen. 
We assume $C=c^{2}>0$ in (4.36). Then, we have the following two cases: For positive constants $a, b$ and $c$,

$$
\left\{\begin{array}{l}
(1) a^{2}\left(u^{\prime}+\nu v\right)^{2}+b^{2}\left(v^{\prime}-\nu u\right)^{2}=c^{2}, \\
(2) a^{2}\left(u^{\prime}+\nu v\right)^{2}-b^{2}\left(v^{\prime}-\nu u\right)^{2}=c^{2} .
\end{array}\right.
$$

LEMmA 4.14. We have $c^{2}=1$ in $(4.36)^{\prime}$.

Proof. (2) We take a function $\theta=\theta(t)$ given by

$$
a\left(u^{\prime}+\nu v\right)=c \cosh \theta, \quad b\left(v^{\prime}-\nu u\right)=c \sinh \theta .
$$

Then, we have

$$
\begin{aligned}
u u^{\prime}+v v^{\prime} & =u\left(\frac{c}{a} \cosh \theta-\nu v\right)+v\left(\frac{c}{b} \sinh \theta+\nu u\right) \\
& =\frac{c}{a} u \cosh \theta+\frac{c}{b} v \sinh \theta
\end{aligned}
$$

Furthermore, since $u=a c \cosh \theta\left(u u^{\prime}+v v^{\prime}\right), v=-b c \sinh \theta\left(u u^{\prime}+v v^{\prime}\right)$ by (4.34), we have

$$
u u^{\prime}+v v^{\prime}=\left(c^{2} \cosh ^{2} \theta-c^{2} \sinh ^{2} \theta\right)\left(u u^{\prime}+v v^{\prime}\right)=c^{2}\left(u u^{\prime}+v v^{\prime}\right) .
$$

Therefore, we have $c^{2}=1$. We take a function $\theta=\theta(t)$ given by $a\left(u^{\prime}+\nu v\right)=$ $c \cos \theta$ and $b\left(v^{\prime}-\nu u\right)=c \sin \theta$ in Case (1). Then, we have also $c^{2}=1$ in the same way to prove Case (2).

The plane curve $(u(t), v(t))$ satisfies the following (1) or (2):

$$
\left\{\begin{array}{l}
(1) a^{2}\left(u^{\prime}+\nu v\right)^{2}+b^{2}\left(v^{\prime}-\nu u\right)^{2}=1, \\
(2) a^{2}\left(u^{\prime}+\nu v\right)^{2}-b^{2}\left(v^{\prime}-\nu u\right)^{2}=1 .
\end{array}\right.
$$

This completes the proof of Proposition 4.8. Moreover, Propositions 4.7 and 4.8 show Theorem 2-(3b). Thus, we complete the proof of Theorem 2 .

We determine plane curves $(u(t), v(t))$ defined by $(4.37)$, that is, we prove Corollaries 2 and 3. First we consider the equation (4.37)-(1). Let $\boldsymbol{e}(s)=(\cos s, \sin s)$ be a parametrized unit circle. We define functions $H=$ $H(s)(>0)$ and $t=t(s)$ by equations

$$
\left\{\begin{array}{l}
\frac{d H}{d s}= \pm H(s) \frac{\sqrt{a^{-2} \cos ^{2} s+b^{-2} \sin ^{2} s}}{\sqrt{1-\left(a^{-2} \cos ^{2} s+b^{-2} \sin ^{2} s\right)}} \\
\frac{d t}{d s}=\mp \frac{H(s)}{\sqrt{1-\left(a^{-2} \cos ^{2} s+b^{-2} \sin ^{2} s\right)}} .
\end{array}\right.
$$


Proposition 4.9. We assume that the set $\left\{t \in I \mid \nu^{\prime}(t)=0\right\}$ does not include any open interval. A curve $(u(t), v(t))$ with $\left(u^{\prime}\right)^{2}+\left(v^{\prime}\right)^{2}=1$ and $\left(u^{\prime \prime}(t), v^{\prime \prime}(t)\right)=\nu(t)\left(-v^{\prime}(t), u^{\prime}(t)\right)$ satisfies the equation $a^{2}\left(u^{\prime}+\nu v\right)^{2}+$ $b^{2}\left(v^{\prime}-\nu u\right)^{2}=1$, if and only if $(u(s), v(s)):=(u(t(s)), v(t(s)))$ is defined by $(u(s), v(s))=H(s) \boldsymbol{e}(s)$ and (4.38), where $s \in \mathbf{R}$ if $b \geq a>1$, and $s \in\left\{s \in \mathbf{R} \mid a^{-2} \cos ^{2} s+b^{-2} \sin ^{2} s<1\right\}$ if $b \geq 1>a>0$.

Proof. (1) We assume $b \geq a>1$. We put $(X, Y)(t)=\left(u^{\prime}+\nu v, v^{\prime}-\right.$ $\nu u)(t)$. For a while, we assume $\nu^{\prime}(t) \neq 0$.
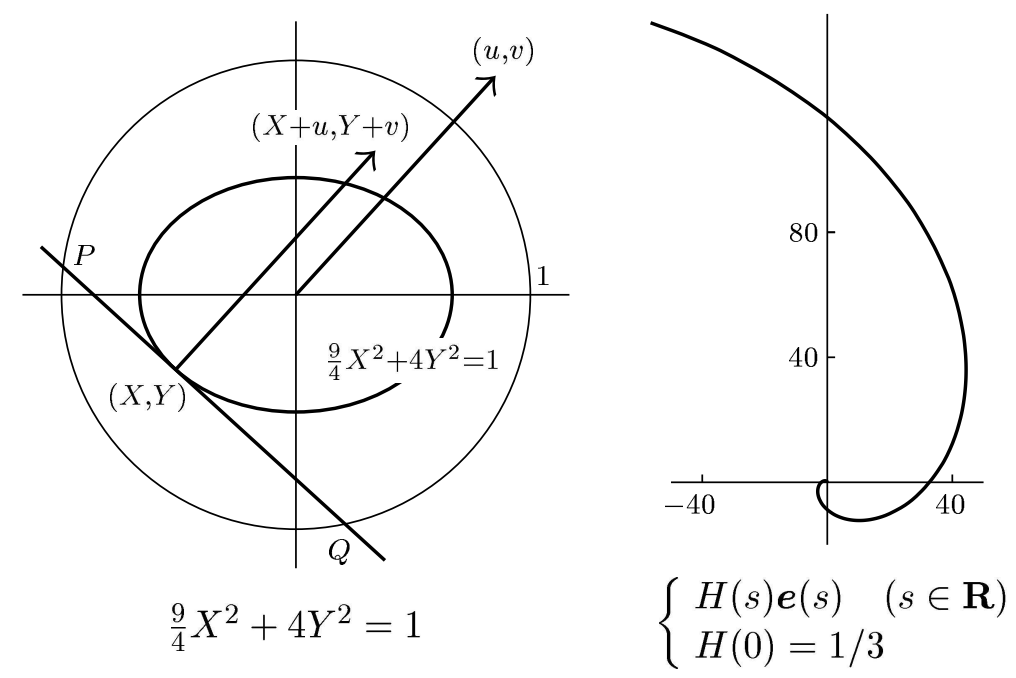

Figure 1.

Then, $\left(X^{\prime}, Y^{\prime}\right)(t)=-\nu^{\prime}(t)(-v, u)(t)$ and $\left(u^{\prime}, v^{\prime}\right)(t)=(X, Y)(t)+$ $\nu(t)(-v, u)(t)$. Thus, we have $\left(u^{\prime}, v^{\prime}\right)(t)=P$ or $Q$ in Figure 1 by $\left(u^{\prime}\right)^{2}+$ $\left(v^{\prime}\right)^{2}=1$. Let $\boldsymbol{e}(t):=\boldsymbol{e}(s(t))=(\cos s(t), \sin s(t))$ be a unit vector field normal to the curve $(X, Y)(t)$. Then, there is a function $H(t)(>0)$ such that $(u, v)(t)=H(t) \boldsymbol{e}(t)$ from $\left(X^{\prime}, Y^{\prime}\right)=-\nu^{\prime}(-v, u)$ and Figure 1 . We replace the parameter $t$ by $s$, which is the parameter of the unit circle. When we take $(d(X, Y) / d s) /\|(d(X, Y) / d s)\|=(\sin s,-\cos s)\left(=: \boldsymbol{e}_{1}(s)\right)$, we have

$$
X(s)=\frac{-a^{-2} \cos s}{\sqrt{a^{-2} \cos ^{2} s+b^{-2} \sin ^{2} s}}, \quad Y(s)=\frac{-b^{-2} \sin s}{\sqrt{a^{-2} \cos ^{2} s+b^{-2} \sin ^{2} s}} .
$$

Indeed, we have

$$
\frac{d}{d s} X=\frac{a^{-2} b^{-2} \sin s}{\left(\sqrt{a^{-2} \cos ^{2} s+b^{-2} \sin ^{2} s}\right)^{3}}, \frac{d}{d s} Y=\frac{-a^{-2} b^{-2} \cos s}{\left(\sqrt{a^{-2} \cos ^{2} s+b^{-2} \sin ^{2} s}\right)^{3}},
$$


and $a^{2} X^{2}(s)+b^{2} Y^{2}(s)=1$. Since $(u(t), v(t))=(u(s(t)), v(s(t))),(X(t)$, $Y(t))=(X(s(t)), Y(s(t)))$ and

$$
\frac{d}{d t}(u(s), v(s))=\frac{d H}{d t} \boldsymbol{e}(s)-H \frac{d s}{d t} \boldsymbol{e}_{1}(s)=(X, Y)(s)+\nu(-v, u)(s),
$$

we have

$$
\left\{\begin{array}{l}
\frac{d H}{d t}=\left\langle\left(\frac{d u}{d t}, \frac{d v}{d t}\right), \boldsymbol{e}(s)\right\rangle=\langle(X, Y)(s), \boldsymbol{e}(s)\rangle+\nu\langle(-v, u)(s), \boldsymbol{e}(s)\rangle \\
=\langle(X, Y)(s), \boldsymbol{e}(s)\rangle=-\sqrt{a^{-2} \cos ^{2} s+b^{-2} \sin ^{2} s} \\
\left(\frac{d H}{d t}\right)^{2}+\left(H \frac{d s}{d t}\right)^{2}=1 .
\end{array}\right.
$$

These equations are quivalent to (4.38).

Since the assumption for $\nu$, there is the open set $J \subset I$ such that $\nu^{\prime}(t) \neq 0$ for $t \in J$. The above argument holds true on $J$. However, since

$$
\nu^{\prime}(t)=\mp \frac{1}{a^{2} b^{2} H^{2}} \frac{\sqrt{1-\left(a^{-2} \cos ^{2} s+b^{-2} \sin ^{2} s\right)}}{\left(\sqrt{a^{-2} \cos ^{2} s+b^{-2} \sin ^{2} s}\right)^{3}}(t)
$$

and continuity of $\nu^{\prime}$, we have $\nu^{\prime}(t) \neq 0$ on $I$, where $I$ is the interval that $H$ is defined. This fact is also true for other curves which we study below.

(2) We assume $b \geq 1>a>0$. Then, the variable $s$ moves on $\{s \mid$ $\left.a^{-2} \cos ^{2} s+b^{-2} \sin ^{2} s<1\right\}$, because $\left(u^{\prime}, v^{\prime}\right)$ does not exist on the unit circle for other $s$ as we see in Figure 2 .

Finally, we consider the equation (4.37)-(2). We define functions $\bar{H}=$ $\bar{H}(s)(>0)$ and $t=t(s)$ by equations

$$
\left\{\begin{array}{l}
\frac{d \bar{H}}{d s}= \pm \frac{\bar{H} \sqrt{a^{-2} \cos ^{2} s-b^{-2} \sin ^{2} s}}{\sqrt{1-\left(a^{-2} \cos ^{2} s-b^{-2} \sin ^{2} s\right)}} \\
\frac{d t}{d s}= \pm \frac{\bar{H}}{\sqrt{1-\left(a^{-2} \cos ^{2} s-b^{-2} \sin ^{2} s\right)}}
\end{array}\right.
$$

where $s \in\left\{s \in(-\pi / 2, \pi / 2) \mid a^{-2} \cos ^{2} s>b^{-2} \sin ^{2} s\right\}$ if $a>1$, and $s \in$ $\left\{s \in(-\pi / 2, \pi / 2) \mid a^{-2} \cos ^{2} s>b^{-2} \sin ^{2} s\right.$ and $\left.a^{-2} \cos ^{2} s-b^{-2} \sin ^{2} s<1\right\}$ if $0<a<1$. 

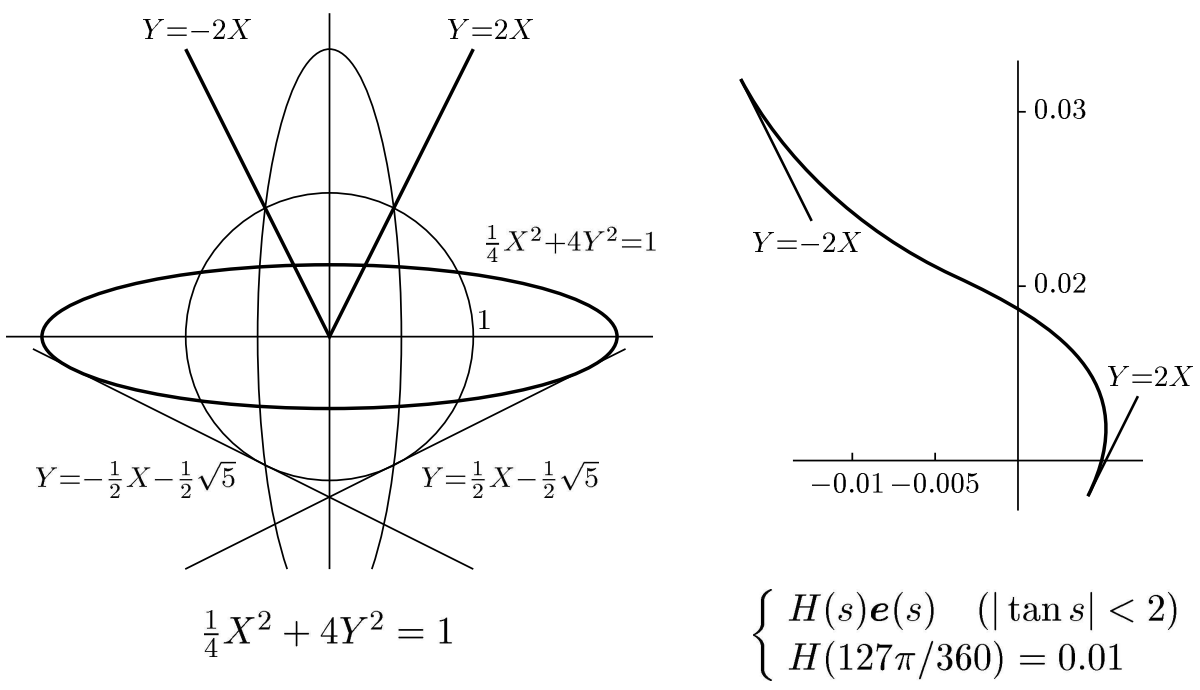

Figure 2.

Proposition 4.10. We assume that the set $\left\{t \in I \mid \nu^{\prime}(t)=0\right\}$ does not include any open interval. A curve $(u(t), v(t))$ with $\left(u^{\prime}\right)^{2}+\left(v^{\prime}\right)^{2}=1$ and $\left(u^{\prime \prime}(t), v^{\prime \prime}(t)\right)=\nu(t)\left(-v^{\prime}(t), u^{\prime}(t)\right)$ satisfies the equation $a^{2}\left(u^{\prime}+\nu v\right)^{2}-$ $b^{2}\left(v^{\prime}-\nu u\right)^{2}=1$, if and only if $(u(s), v(s)):=(u(t(s)), v(t(s)))$ is defined by $(u(s), v(s))=\bar{H}(s) \boldsymbol{e}(s)$ and $(4.39)$.

Proof. (1) We assume $a>1$. We put $(X, Y)(t)=\left(u^{\prime}+\nu v, v^{\prime}-\right.$ $\nu u)(t)$. Then, $\left(X^{\prime}, Y^{\prime}\right)(t)=-\nu^{\prime}(t)(-v, u)(t)$ and $\left(u^{\prime}, v^{\prime}\right)(t)=(X, Y)(t)+$ $\nu(t)(-v, u)(t)$.

Thus we have $\left(u^{\prime}, v^{\prime}\right)(t)=P^{\prime}$ or $Q^{\prime}$ in Figure 3. Let $\boldsymbol{e}(t):=\boldsymbol{e}(s(t))=$ $(\cos s(t), \sin s(t))$ be a unit vector field normal to the curve $(X, Y)(t)$. Then, there exists a function $\bar{H}(t)(>0)$ such that $(u, v)(t)=\bar{H}(t) \boldsymbol{e}(t)$ from $\left(X^{\prime}, Y^{\prime}\right)=-\nu^{\prime}(-v, u)$ and Figure 3 . We replace the parameter $t$ by $s$. When we take $d(X, Y) / d s /\|d(X, Y) / d s\|=(\sin s,-\cos s)\left(=: \boldsymbol{e}_{1}(s)\right)$, we have

$$
\begin{aligned}
& X(s)=\frac{\cos s}{a^{2} \sqrt{a^{-2} \cos ^{2} s-b^{-2} \sin ^{2} s}} \\
& Y(s)=-\frac{\sin s}{b^{2} \sqrt{a^{-2} \cos ^{2} s-b^{-2} \sin ^{2} s}}
\end{aligned}
$$



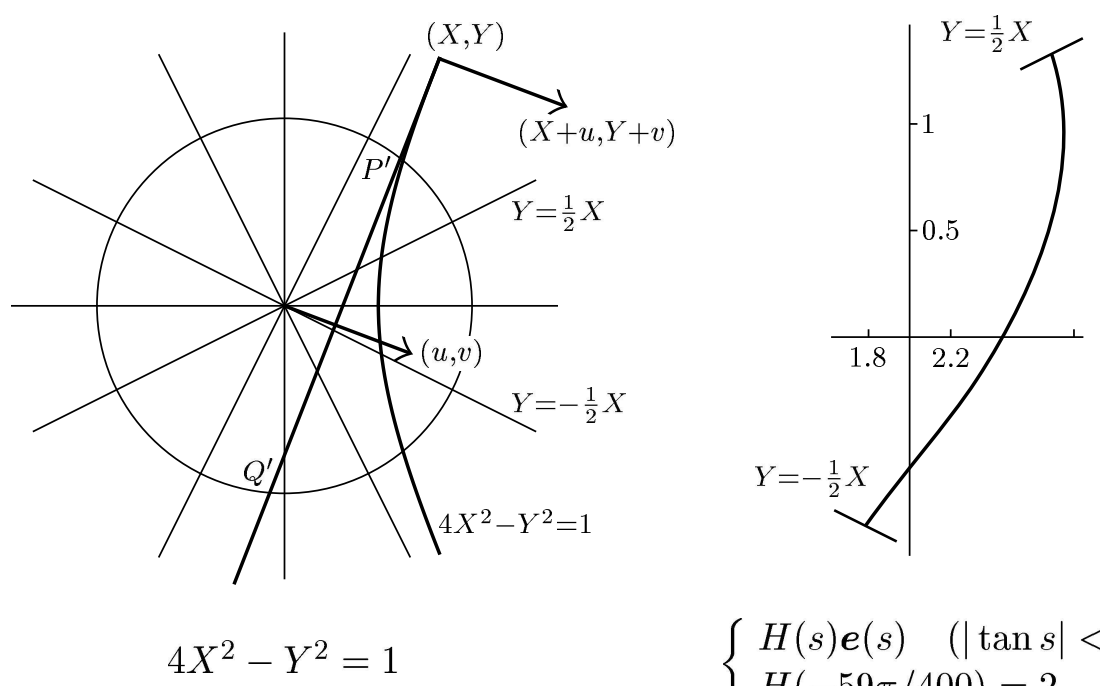

Figure 3.

$\left\{\begin{array}{l}H(s) \boldsymbol{e}(s) \quad(|\tan s|<1 / 2) \\ H(-59 \pi / 400)=2\end{array}\right.$

Figure 3.

Indeed, we have

$$
\begin{aligned}
\frac{d}{d s} X & =\frac{\sin s}{a^{2} b^{2}\left(\sqrt{a^{-2} \cos ^{2} s-b^{-2} \sin ^{2} s}\right)^{3}} \\
\frac{d}{d s} Y & =-\frac{\cos s}{a^{2} b^{2}\left(\sqrt{a^{-2} \cos ^{2} s-b^{-2} \sin ^{2} s}\right)^{3}}
\end{aligned}
$$

and $a^{2} X^{2}-b^{2} Y^{2}=1$. Since $(u(t), v(t))=(u(s(t)), v(s(t))),(X(t), Y(t))=$ $(X(s(t)), Y(s(t)))$ and

$$
\frac{d}{d t}(u(s), v(s))=\frac{d \bar{H}}{d t} e(s)-\bar{H} \frac{d s}{d t} \boldsymbol{e}_{1}(s)(=(X, Y)(s)+\nu(-v, u)(s)),
$$

we have

$$
\left\{\begin{aligned}
& \frac{d \bar{H}}{d t}=\left\langle\left(\frac{d u}{d t}, \frac{d v}{d t}\right), e(s)\right\rangle=\langle(X, Y)(s), e(s)\rangle \\
&=\sqrt{a^{-2} \cos ^{2} s-b^{-2} \sin ^{2} s} \\
&\left(\frac{d \bar{H}}{d t}\right)^{2}+\left(\bar{H} \frac{d s}{d t}\right)^{2}=1 .
\end{aligned}\right.
$$


If $|\tan s|<b / a$ and $s \in(-\pi / 2, \pi / 2), \boldsymbol{e}(s)$ becomes the vector field normal to $(X, Y)(s)$.

(2) If $0<a<1$, then $\left(u^{\prime}, v^{\prime}\right)(t)$ exists on the unit circle only for $s \in\left\{s \in(-\pi / 2, \pi / 2)|| \tan s \mid<b / a\right.$ and $\left.a^{-2} \cos ^{2} s-b^{-2} \sin ^{2} s<1\right\}$ as we see in Figure 4.

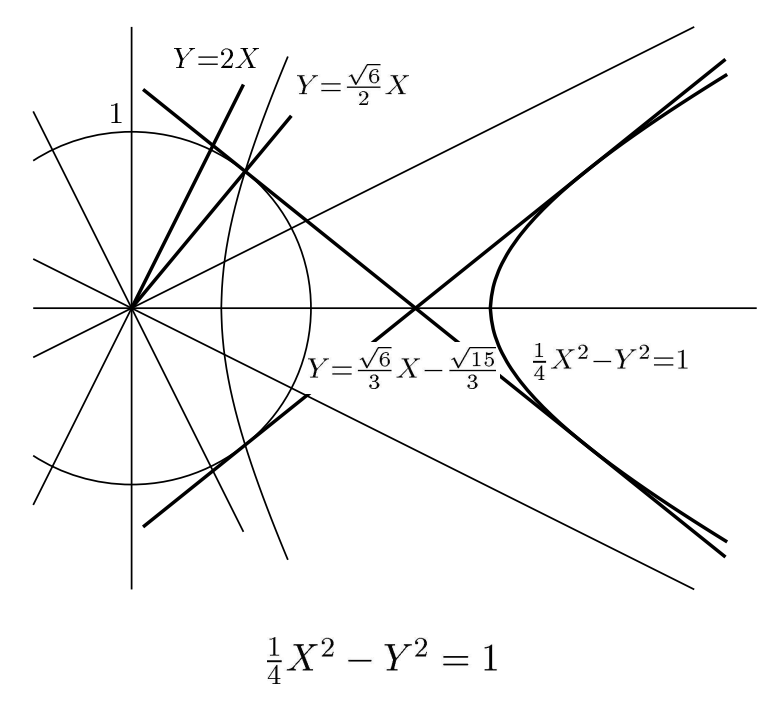

Figure 4.

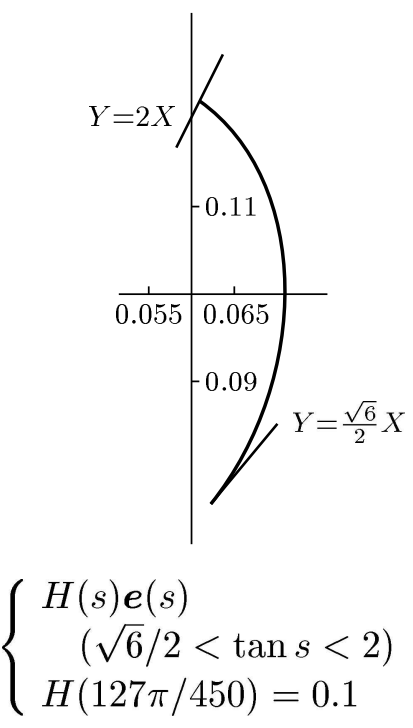

\section{REFERENCES}

[1] E. Cartan, La déformation des hypersurfaces dans L'espace conforme á $n \geq 5$ dimensions, Oeuvres complétes III, 1, pp. 221-286.

[2] U. Hertrich-Jeromin, On conformally flat hypersurfaces and Guichard's nets, Beitr. Alg. Geom., 35 (1994), 315-331.

[3] J. Lafontaine, Conformal geometry from Riemannian viewpoint, Conformal Geometry (R. S. Kulkarni and U. Pinkall, eds.), Aspects of Math. Vol. E12, Max-Plank-Ins. für Math. (1988), pp. 65-92.

[4] G. M. Lancaster, Canonical metrics for certain conformally Euclidean spaces of dimension three and codimension one, Duke Math. J., 40 (1973), 1-8.

[5] Y. Suyama, Explicit representation of compact conformally flat hypersurfaces, Tôhoku Math. J., 50 (1998), 179-196. 
Department of Applied Mathematics Fukuoka University

Fukuoka, 814-0180

Japan

suyama@fukuoka-u.ac.jp 